\title{
Poverty trap and global indeterminacy in a growth model with open-access natural resources
}

\author{
Angelo Antoci ${ }^{\mathrm{a}, *}$, Marcello Galeotti ${ }^{\mathrm{b}}$, Paolo Russu ${ }^{\mathrm{a}}$ \\ a Dipartimento di Economia Impresa e Regolamentazione, Università di Sassari, via Torre Tonda 34, \\ 07100 Sassari, Italy \\ ${ }^{\mathrm{b}}$ Dipartimento di Matematica per le Decisioni, Università di Firenze, via Lombroso 6/17, 50134 Firenze, Italy
}

Received 16 June 2009; final version received 21 September 2010; accepted 27 September 2010

Available online 3 December 2010

\begin{abstract}
In this paper we use global analysis techniques to analyze an economic growth model with environmental negative externalities, giving rise to a three-dimensional dynamic system (the framework is the one introduced by Wirl (1997) [53]). The dynamics of our model admits a locally attracting stationary state $P_{1}^{*}$, which is, in fact, a poverty trap, coexisting with another stationary state $P_{2}^{*}$ possessing saddle-point stability. Global dynamical analysis shows that, under some conditions on the parameters, if the initial values of the state variables are close enough to the coordinates of $P_{1}^{*}$, then there exists a continuum of equilibrium trajectories approaching $P_{1}^{*}$ and one trajectory approaching $P_{2}^{*}$. Therefore, our model exhibits global indeterminacy, since either $P_{1}^{*}$ or $P_{2}^{*}$ can be selected according to agent expectations. Moreover, we prove that conditions guaranteeing the attractivity of $P_{1}^{*}$ also imply the saddle-point stability of $P_{2}^{*}$. However, when $P_{1}^{*}$ is not attractive, numerical simulations show the possible existence of one or two limit cycles: an attractive one surrounding $P_{1}^{*}$ and one endowed with a two-dimensional stable manifold surrounding $P_{2}^{*}$. (c) 2010 Elsevier Inc. All rights reserved.
\end{abstract}

JEL classification: C62; O13; O41; Q22

Keywords: Global and local indeterminacy; Poverty trap; Environmental externalities; History versus expectations; Open-access natural resources

\footnotetext{
The authors wish to thank Tadashi Shigoka for his precious comments to a preliminary version of the article together with an associate editor and two referees of this journal, whose remarks and suggestions have allowed a substantial improvement of the work. The research of Angelo Antoci has been supported by a grant of the Fondazione Banco di Sardegna and that of Marcello Galeotti by a grant of the Istituto Nazionale di Alta Matematica Francesco Severi.

* Corresponding author.

E-mail address: angelo.antoci@virgilio.it (A. Antoci).
}

0022-0531/\$ - see front matter @ 2010 Elsevier Inc. All rights reserved. doi:10.1016/j.jet.2010.12.003 


\section{Introduction}

As Krugman [31] and Matsuyama [35] pointed out in their seminal papers, equilibrium selection in dynamic optimization models with externalities may depend on expectations; that is, given the initial values of the state variables (history), the path followed by the economy may be determined by the choice of the initial values of the jumping variables. This implies that expectations play a key role in equilibrium selection and in fact global indeterminacy may occur: that is, starting from the same initial values of the state variables, different equilibrium paths can approach different $\omega$-limit sets (for example, different stationary states). In this context, local stability analysis may be misleading, in that it refers to a neighborhood of a stationary state, whereas the initial values of jumping variables do not have to belong to such a neighborhood. In the words of Matsuyama [35, p. 619]:

"Knowing the local dynamics is not enough, because, for example, demonstrating the uniqueness of the perfect foresight path in a neighborhood of a stationary state does not necessarily rule out the existence of other perfect foresight paths in the large."

Although some works on indeterminacy focus on global dynamics and stress the relevance of global analysis (see, among the others, Pintus et al. [45], Raurich-Puigdevall [46], Benhabib and Eusepi [11], Karp and Paul [30], Pérez and Ruiz [43], Benhabib et al. [12], Mattana et al. [36], Coury and Wen [22], Brito and Venditti [17], Antoci et al. [4]), the literature on indeterminacy is almost exclusively based on local analysis, due to the fact that dynamic models exhibiting indeterminacy are often highly nonlinear and difficult to be analyzed globally. ${ }^{1}$ Few papers study global indeterminacy in environmental dynamics; Karp and Paul [30] study (two-dimensional) dynamics of labor migration and environmental change in an economy in which there are two productive sectors, both generating pollution but only one affected by it; Pérez and Ruiz [43] find that global indeterminacy can occur in a two-dimensional endogenous growth model with pollution and public abatement activities; Antoci et al. [4], in an overlapping generations context, show that the consumption of private goods as substitutes for free-access environmental goods can produce global indeterminacy and chaos.

The objective of our paper is to highlight the relevance of global indeterminacy in a context in which economic activity depends on the exploitation of a free-access natural resource. We analyze a growth model with environmental externalities, giving rise to a three-dimensional nonlinear dynamic system (the framework is that introduced by Wirl [53]). ${ }^{2}$ In particular, we study

\footnotetext{
1 In the present work we are not dealing with another important problem in economic dynamics, namely the existence of indifference points in an optimal control problem. Starting from these points, several optimal solutions exist, giving rise to the same value of the objective function (see the seminal contributions of Sethi [48], Skiba [49], Dechert and Nishimura [24] and, among the others, the recent works of Hartl et al. [27], Caulkins et al. [19,20], Wirl and Feichtinger [55], Wagener [50,51]). Vice versa, in our context, as it happens to be the case in the literature on indeterminacy, the trajectories followed by the economy are Nash equilibria but do not represent optimal solutions, being the dynamics conditioned by externalities. Therefore, when multiple Nash equilibrium trajectories exist, starting from the same initial values of the state variables, economic agents may select one which is Pareto-dominated by others, due to coordination problems.

2 Wirl analyzes dynamic optimization problems of competitive agents with one state variable $x$ and one dynamic externality $y$. Whereas the time evolution of $y$ is affected by agents' choices, the impact due to each single agent is negligible; consequently, the evolution of $y$ is considered as an exogenous datum. Such optimization problems lead to three-dimensional dynamic systems. The work of Wirl focuses on local stability properties of stationary states and on the occurrence of Hopf bifurcations in this class of systems.
} 
the equilibrium growth dynamics of an economy constituted by a continuum of identical agents. At each instant of time $t$, the representative agent produces the output $Y(t)$ by labor $L(t)$, by the accumulated physical capital $K(t)$ and by the stock $E(t)$ of an open-access renewable natural resource. The economy-wide aggregate production $\bar{Y}(t)$ negatively affects the stock of the natural resource; however, the value of $\bar{Y}(t)$ is considered as exogenously determined by the representative agent, so that economic dynamics is affected by negative environmental externalities. ${ }^{3}$

We assume that the representative agent's instantaneous utility, depending on leisure $1-L(t)$ and consumption $C(t)$ of the output $Y(t)$, is represented by the additively non-separable function $\frac{\left[C(1-L)^{\varepsilon}\right]^{1-\eta}-1}{1-\eta}$. Moreover, we assume that the production technology is represented by the CobbDouglas function $[K(t)]^{\alpha}[L(t)]^{\beta}[E(t)]^{\gamma}$, with $\alpha+\beta<1$ and $\alpha, \beta, \gamma>0$.

In this context, we show that, if $\alpha+\gamma<1$, the dynamics can admit a locally attracting stationary state $P_{1}^{*}=\left(K_{1}^{*}, E_{1}^{*}, L^{*}\right)$, in fact a poverty trap, coexisting with another stationary state $P_{2}^{*}=\left(K_{2}^{*}, E_{2}^{*}, L^{*}\right)$, where $K_{1}^{*}<K_{2}^{*}$ and $E_{1}^{*}<E_{2}^{*}$, possessing saddle-point stability.

Global analysis shows that, under some conditions on the parameters, if the economy starts from initial values $K_{0}$ and $E_{0}$ sufficiently close to $K_{1}^{*}$ and $E_{1}^{*}$, then there exists a continuum of initial values $L_{0}^{1}$ such that the trajectory from $\left(K_{0}, E_{0}, L_{0}^{1}\right)$ approaches $P_{1}^{*}$ and a locally unique initial value $L_{0}^{2}$ such that the trajectory from $\left(K_{0}, E_{0}, L_{0}^{2}\right)$ approaches $P_{2}^{*}$ (see Fig. 6). ${ }^{4}$ Therefore our model exhibits local indeterminacy (i.e. there exists a continuum of trajectories leading to $P_{1}^{*}$ ), but also global indeterminacy, since either $P_{1}^{*}$ or $P_{2}^{*}$ may be selected according to agents' expectations. Along the trajectories belonging to the basin of attraction of $P_{1}^{*}$, over-exploitation of the natural resource drives the economy towards a tragedy of commons scenario.

These results are obtained through a partial description of the shape of the saddle-point twodimensional stable manifold. A full description of such a manifold is usually very difficult or impossible. However, in a three-dimensional system, two-dimensional stable (or unstable) manifolds are separatrices between different regimes of the trajectories. Therefore, if one is able to detect, in a significant region of the phase space, a separatrix between two sets of points whose trajectories show different behavior, that may lead to relevant information on the manifold of interest. We observe that this approach, which in some contexts, as the present one, can prove useful, differs from others in global indeterminacy literature, based on bifurcation techniques; see, for example, Matsuyama [35], Pintus et al. [45], Benhabib and Eusepi [11], Benhabib et al. [12], Mattana et al. [36]. However it must be stressed that gaining information on separatrices is paramount to any global analysis: in particular it can lead to information on size and/or shape of attractive basins (which several authors consider the main goal of global indeterminacy analysis). ${ }^{5}$

\footnotetext{
3 Environmental externalities can affect economic activities especially in developing countries, where property rights tend to be ill-defined and ill-protected, environmental institutions and regulations are weak and natural resources are more fragile than in developed countries, which are located in temperate areas instead than in tropical and sub-tropical regions (see e.g. López [32,33]).

4 In Krugman's model global indeterminacy occurs only when the economy starts sufficiently away from both stationary states. On the other hand, in Matsuyama's model global indeterminacy occurs even if the economy starts in the neighborhood of the poverty trap; so our model dynamics is much closer to Matsuyama's than to Krugman's.

5 For example Wirl [54] analyzes a separatrix problem in an optimal growth model where, differently from our case, the only production factor is physical capital $K$ and a renewable environmental resource $R$ enters only into the utility function. The analytical context is also quite different from ours. In fact Wirl considers optimal solutions for a fourdimensional system exhibiting two saddle-point stable stationary states. It turns out that in the two-dimensional state space $(K, R)$ the basins of attraction of the two stable stationary states are separated by a curve, whose corresponding
} 
Our paper also contains some numerical simulations, which provide further insights about the dynamics of the model:

(1) Global indeterminacy may occur also in the context in which $P_{1}^{*}$ has a stable manifold of dimension one and $P_{2}^{*}$ is either saddle-point stable or repelling (three positive real part eigenvalues), and thus no locally indeterminate stationary state exists. In such a context, numerical simulations show that, in case $P_{2}^{*}$ is a saddle-point, then, starting from the same initial values of $K$ and $E$, the economy may approach either the determinate stationary state $P_{2}^{*}$ or an attracting limit cycle surrounding $P_{1}^{*}$ (see Fig. 8). Vice versa, when $P_{2}^{*}$ is a source (i.e. repelling), simulations indicate the possible existence of two limit cycles (see Fig. 9), so that, starting from the same initial values of $K$ and $E$, the economy may approach either an attracting limit cycle surrounding $P_{1}^{*}$ or a limit cycle endowed with a two-dimensional stable manifold surrounding $P_{2}^{*}$.

(2) Numerical simulations suggest that the stable manifold of the locally determinate point $P_{2}^{*}$ bounds the basin of attraction of the locally indeterminate point $P_{1}^{*}$ or of the attracting cycle around $P_{1}^{*}$ (see Figs. 6, 8). This implies that even if the economy starts very close to the locally determinate point $P_{2}^{*}$, it can move quite far away from it, in particular toward a poverty trap.

Properties (1) and (2) are analogous to those obtained in Coury and Wen [22] and in Brito and Venditti [17]. Coury and Wen study a real business cycle model in discrete time, where there exists a unique stationary state, locally determinate (i.e. saddle-point stable), "surrounded" by stable periodic trajectories, so that the economy can approach either the stationary state or the periodic trajectories, starting from the same initial values of the state variables. Brito and Venditti analyze a two-sector growth model with accumulation of human and physical capital that admits two balanced growth paths (BGPs). They find that global indeterminacy can occur in the following contexts: (a) both BGPs are locally determinate; (b) both BGPs are locally indeterminate; (c) one BGP is locally indeterminate (the poverty trap) while the other is determinate.

Our analysis focuses on global indeterminacy of dynamics, but it also gives sufficient conditions for local indeterminacy. There exists an enormous literature on local indeterminacy in economic growth models. Although in this article we don't have room for a review, we point out the place that our results occupy in the current research. In fact, even if the main body of the literature on local indeterminacy concerns economies with increasing social returns, ${ }^{6}$ a growing proportion of articles deals with models where indeterminacy is obtained under the assumption of

optimal trajectories lie on the one-dimensional stable manifold of a third conditionally stable stationary point. Moreover, Wirl shows the possible existence of limit cycles around the stationary states, arisen through Hopf bifurcations. Vice versa, we analyze a three-dimensional system whose trajectories are sub-optimal Nash solutions of a dynamical control problem. In such a context the two-dimensional stable manifold of a saddle-point stable stationary state may separate, in the three-dimensional phase space, the basin of attraction of another Pareto-dominated stationary state (a poverty trap) from a region whose trajectories tend to a boundary point where the economy collapses (i.e. physical capital and labor tend to zero, while the environmental resource tends to its carrying capacity). The possible existence of limit cycles, generated by Hopf bifurcations, is shown also in our case, through numerical simulations.

6 See, for example, early studies of Benhabib and Farmer [7] and Boldrin and Rustichini [16] where the degree of increasing returns is assumed sufficiently large to produce indeterminacy. Subsequent works have shown that indeterminacy may also emerge without assuming strong degree of increasing returns; see, among the others, Perli [44], Bennett and Farmer [13], Nishimura et al. [42]. For a review of the literature on local indeterminacy in models with externalities see Benhabib and Farmer [10] and Mino et al. [40]. 
social constant return technologies; see, for example, Benhabib and Nishimura [8,9], Mino [39], Nishimura and Shimomura [41], Zhang [56], Mino et al. [40], Brito and Venditti [17]. In our paper, local indeterminacy can occur with social constant or decreasing returns and is generated by negative environmental externalities of production activity affecting the natural resource. Other works focus on the role played by negative externalities in producing local indeterminacy. For example, Chen and Lee [21] consider a social constant returns economy where a congestible public good exerts positive sector-specific externalities, while a congestion effect generates negative aggregate externalities. Itaya [29] shows how pollution may affect indeterminacy results in a one-sector growth model with social increasing returns. In Meng and Yip [38] indeterminacy is produced by negative capital externalities. In Antoci et al. [1] and in Antoci and Sodini [2] negative externalities may generate indeterminacy in an economy where private goods can be consumed as substitutes for free-access environmental goods.

The present paper has the following structure. Sections 2 and 3 define the set-up of the model and the associated dynamic system. Section 4 deals with the existence and local stability of stationary states and with Hopf bifurcations arising from stability changes. Section 5 is devoted to the global analysis of dynamics and provides the main results of the paper. Mathematical Appendices A (proof of Theorem 4) and B (proof of Lemma 7) conclude the paper.

\section{Set-up of the model}

The economy we analyze is constituted by a continuum of identical economic agents; the size of the population of agents is normalized to unity. At each instant of time $t \in[0, \infty)$, the representative agent produces an output $Y(t)$ by the following Cobb-Douglas technology

$$
Y(t)=[K(t)]^{\alpha}[L(t)]^{\beta}[E(t)]^{\gamma}, \quad \text { with } \alpha+\beta<1 \text { and } \alpha, \beta, \gamma>0
$$

where $K(t)$ is the stock of physical capital accumulated by the representative agent, $L(t)$ is the agent's labor input and $E(t)$ is the stock of an open-access renewable natural resource. ${ }^{7} \mathrm{We}$ assume that the representative agent's instantaneous utility function depends on leisure $1-L(t)$ and consumption $C(t)$ of the output $Y(t)$; precisely, we consider the following additively nonseparable function (a function of this type is used, among the others, by Bennet and Farmer [13], Mino [39] and Itaya [29])

$$
U[C(t), L(t)]=\frac{\left[C(t)(1-L(t))^{\varepsilon}\right]^{1-\eta}-1}{1-\eta}
$$

where $\varepsilon, \eta>0$ and $\eta \neq 1$. Moreover, we assume that the utility function is concave in $C$ and in $1-L$, i.e. $\eta>\frac{\varepsilon}{1+\varepsilon}$. The parameter $\eta$ denotes the inverse of the intertemporal elasticity of substitution in consumption. Our function displays a constant intertemporal elasticity of substitution and possesses the property that income and substitution effects exactly balance each other in the labor supply equation.

\footnotetext{
7 In modeling production activity based on open-access natural resources (for example, fishery, forestry and tourism), the stock $E(t)$ of the environmental resource very often enters as an input in the production function: see, for example, Berck and Perloff [14], Ayong Le Kama [6], López et al. [34]. Some authors use the Cobb-Douglas production function introduced by Gordon [26] and Schaefer [47], with all the exponents equal to one (see, e.g., López et al. [34]). We have chosen, instead, to work with a more general Cobb-Douglas function, allowing to analyze the case with constant social returns to scale.
} 
The evolution of $K(t)$ (assuming, for simplicity, the depreciation of $K$ to be zero) is represented by the differential equation

$$
\dot{K}=K^{\alpha} L^{\beta} E^{\gamma}-C
$$

where $\dot{K}$ is the time derivative of $K$. In order to model the dynamics of $E$ we start from the well-known logistic equation ${ }^{8}$ and augment it by considering the negative impact due to the production process

$$
\dot{E}=E(\bar{E}-E)-\delta \bar{Y}
$$

where the parameter $\bar{E}>0$ represents the carrying capacity of the natural resource, $\bar{Y}$ is the economy-wide average output and the parameter $\delta>0$ measures the negative impact of $\bar{Y}$ on $E$. ${ }^{9}$ Under the specification (1) of the environmental dynamics, the production process in our economy can be interpreted as an extractive activity. Its impact on the natural resource is given by the rate of harvest which is proportional to $\bar{Y}$. This assumption is usual in models of economic dynamics depending on open-access resources (see, for example, Berck and Perloff [14], Wirl [52], Bischi and Lamantia [15], D'Alessandro [23], McWhinnie [37]) and has been also introduced in economic growth models where a natural resource-intensive sector is considered (see, for example, Ayong Le Kama [6] and López et al. [34]).

We assume that the representative agent chooses the functions $C(t)$ and $L(t)$ in order to solve the following problem

$$
\underset{C, L}{\operatorname{MAX}} \int_{0}^{\infty} \frac{\left[C(1-L)^{\varepsilon}\right]^{1-\eta}-1}{1-\eta} e^{-\theta t} d t
$$

subject to

$$
\begin{aligned}
& \dot{K}=K^{\alpha} L^{\beta} E^{\gamma}-C \\
& \dot{E}=E(\bar{E}-E)-\delta \bar{Y}
\end{aligned}
$$

with $K(0)$ and $E(0)$ given, $K(t), E(t), C(t) \geqslant 0$ and $1 \geqslant L(t) \geqslant 0$ for every $t \in[0,+\infty) ; \theta>0$ is the discount rate.

We assume that capital $K$ is reversible, i.e., we allow for disinvestment $(\dot{K}<0)$ at some instants of time.$^{10}$ Furthermore we assume that, in solving problem (2), the representative agent considers $\bar{Y}$ as exogenously determined since, being economic agents a continuum, the impact on $\bar{Y}$ of each one is null. However, since agents are identical, ex post $\bar{Y}=Y$ holds. This implies that the trajectories resulting from our model are not socially optimal but Nash equilibria, because no agent has an incentive to modify his choices if the others don't modify theirs.

\section{Dynamics}

The current value Hamiltonian function associated to problem (2) is (see Wirl [53])

\footnotetext{
8 The logistic function has been extensively used as a growth function of renewable resources; see, for example, Brown [18] and Elíasson and Turnovsky [25].

${ }^{9}$ Notice that $\bar{E}$ is the value that $E$ would reach, as $t \rightarrow+\infty$, in absence of the negative impact due to economic activity.

10 This amounts to assume that the economy we are analyzing is a small open economy that can sell or buy capital goods abroad at a fixed price.
} 


$$
H=\frac{\left[C(1-L)^{\varepsilon}\right]^{1-\eta}-1}{1-\eta}+\Omega\left(K^{\alpha} L^{\beta} E^{\gamma}-C\right)
$$

where $\Omega$ is the co-state variable associated to $K$. By applying the Maximum Principle, the dynamics of the economy is described by the system

$$
\begin{aligned}
& \dot{K}=\frac{\partial H}{\partial \Omega}=K^{\alpha} L^{\beta} E^{\gamma}-C \\
& \dot{\Omega}=\theta \Omega-\frac{\partial H}{\partial K}=\Omega\left(\theta-\alpha K^{\alpha-1} L^{\beta} E^{\gamma}\right)
\end{aligned}
$$

with the constraint

$$
\dot{E}=E(\bar{E}-E)-\delta \bar{Y}
$$

where $C$ and $L$ satisfy the following conditions ${ }^{11}$

$$
\begin{aligned}
& \frac{\partial H}{\partial C}=C^{-\eta}(1-L)^{\varepsilon(1-\eta)}-\Omega=0 \\
& \frac{\partial H}{\partial L}=0, \quad \text { i.e. } \beta(1-L) \Omega K^{\alpha} L^{\beta-1} E^{\gamma}-\varepsilon C^{1-\eta}(1-L)^{\varepsilon(1-\eta)}=0
\end{aligned}
$$

Since our system meets the Mangasarian hypotheses, the above conditions plus the limit transversality condition $\lim _{t \rightarrow+\infty} \Omega(t) K(t) e^{-\theta t}=0$ are sufficient for solving problem (2). This is the case also if $\alpha+\beta+\gamma>1$ (remember we assumed $\alpha+\beta<1$ ), because the stock $E$ is considered as a positive externality in the decision problem of the representative agent.

By replacing $\bar{Y}$ with $K^{\alpha} L^{\beta} E^{\gamma}$, the Maximum Principle conditions yield a dynamic system with two state variables, $K$ and $E$, and one jumping variable, $\Omega$. Notice that, from $\varepsilon C \frac{\partial H}{\partial C}+$ $\frac{\partial H}{\partial L}=0$, one obtains

$$
\begin{aligned}
& C=\frac{\beta}{\varepsilon}(1-L) L^{\beta-1} K^{\alpha} E^{\gamma} \\
& f(L):=\frac{\varepsilon}{\beta}(1-L)^{\frac{\varepsilon-\eta(1+\varepsilon)}{\eta}} L^{1-\beta}=K^{\alpha} E^{\gamma} \Omega^{\frac{1}{\eta}}
\end{aligned}
$$

Hence one can write the following system, equivalent to (3)-(4)

$$
\begin{aligned}
\dot{K}= & \frac{1}{\varepsilon} K^{\alpha} E^{\gamma} L^{\beta-1}[(\beta+\varepsilon) L-\beta] \\
\dot{E}= & E(\bar{E}-E)-\delta K^{\alpha} L^{\beta} E^{\gamma} \\
\dot{L}= & \frac{f(L)}{f^{\prime}(L)}\left[\frac{\alpha}{\varepsilon} K^{\alpha-1} E^{\gamma} L^{\beta-1}[(\beta+\varepsilon) L-\beta]+\gamma\left(\bar{E}-E-\delta K^{\alpha} L^{\beta} E^{\gamma-1}\right)\right. \\
& \left.+\frac{1}{\eta}\left(\theta-\alpha K^{\alpha-1} L^{\beta} E^{\gamma}\right)\right]
\end{aligned}
$$

In such a context, the jumping variable is $L$, instead of $\Omega$ ( $L$ and $\Omega$ are related by (5)). As a consequence, given the initial values of the state variables, $K_{0}$ and $E_{0}$, the representative agent has to choose the initial value $L_{0}$ of $L$.

\footnotetext{
$\overline{11}$ Notice that the utility function we adopted implies $C>0$ and $0<L<1$.
} 


\section{Stationary states, stability and Hopf bifurcations}

We recall the conditions on the parameters: they are all positive, with $\alpha+\beta<1$ and $1 \neq \eta>$ $\frac{\varepsilon}{1+\varepsilon}$. The following theorem deals with the problem of the existence and numerosity of stationary states of the dynamic system (6).

Theorem 1. System (6) has one stationary state if $\alpha+\gamma>1$; one or zero stationary states if $\alpha+\gamma=1$; zero, one or two stationary states if $\alpha+\gamma<1$.

Proof. A stationary state $P^{*}=\left(K^{*}, E^{*}, L^{*}\right)$ of (6) must satisfy

$$
\begin{aligned}
& L^{*}=\frac{\beta}{\beta+\varepsilon} \\
& K^{*}=\frac{\alpha}{\delta \theta} E^{*}\left(\bar{E}-E^{*}\right) \\
& g\left(E^{*}\right):=E^{*}+\delta\left(\frac{\beta}{\beta+\varepsilon}\right)^{\frac{\beta}{1-\alpha}}\left(\frac{\alpha}{\theta}\right)^{\frac{\alpha}{1-\alpha}}\left(E^{*}\right)^{\frac{\alpha+\gamma-1}{1-\alpha}}=\bar{E}
\end{aligned}
$$

i.e.

$$
\begin{aligned}
& L^{*}=\frac{\beta}{\beta+\varepsilon} \\
& K^{*}=\left(\frac{\beta}{\beta+\varepsilon}\right)^{\frac{\beta}{1-\alpha}}\left(\frac{\alpha}{\theta}\right)^{\frac{1}{1-\alpha}}\left(E^{*}\right)^{\frac{\gamma}{1-\alpha}} \\
& g\left(E^{*}\right):=E^{*}+\delta\left(\frac{\beta}{\beta+\varepsilon}\right)^{\frac{\beta}{1-\alpha}}\left(\frac{\alpha}{\theta}\right)^{\frac{\alpha}{1-\alpha}}\left(E^{*}\right)^{\frac{\alpha+\gamma-1}{1-\alpha}}=\bar{E}
\end{aligned}
$$

Hence the graph of $g(E)$ intersects the line $E=\bar{E}$ exactly at one point if $\alpha+\gamma>1$, at most at one point if $\alpha+\gamma=1$, at zero, one or two points if $\alpha+\gamma<1$, while $K^{*}$ is an increasing function of $E^{*}$ (see Fig. 1).

Observe that, if $\alpha+\gamma<1$, then there exists one stationary state only if the minimum of the function $g\left(E^{*}\right)$ coincides with the value $\bar{E}$; so, generically, the stationary states are zero or two (see Fig. 1). By (8), when two stationary states exist, $P_{1}^{*}=\left(K_{1}^{*}, E_{1}^{*}, L^{*}\right)$ and $P_{2}^{*}=\left(K_{2}^{*}, E_{2}^{*}, L^{*}\right)$, then $K_{1}^{*}<K_{2}^{*}$ and $E_{1}^{*}<E_{2}^{*}$; so $P_{2}^{*}$ Pareto-dominates $P_{1}^{*}$. If the economy approaches the latter, then a tragedy of commons scenario emerges, characterized by over-exploitation of the natural resource and by low physical capital accumulation (labor input is equal to $L^{*}=\frac{\beta}{\beta+\varepsilon}$ at both stationary states). ${ }^{12}$ Notice that, in our model, multiplicity of stationary states may occur also in a context of social constant returns to scale, $\alpha+\beta+\gamma=1$, whereas it is ruled out if the elasticity $\gamma$ of the production function with respect to natural capital $E$ is relatively high, that is if $\alpha+\gamma \geqslant 1$.

Now, let $P^{*}=\left(K^{*}, E^{*}, L^{*}\right)$ be a stationary state of (6) and consider the Jacobian matrix of system (6) evaluated at $P^{*}$

\footnotetext{
12 It is worth to stress that, even if a trajectory approaches $P_{2}^{*}$, it does not represent an optimal growth path, since environmental externalities are not internalized by economic agents.
} 


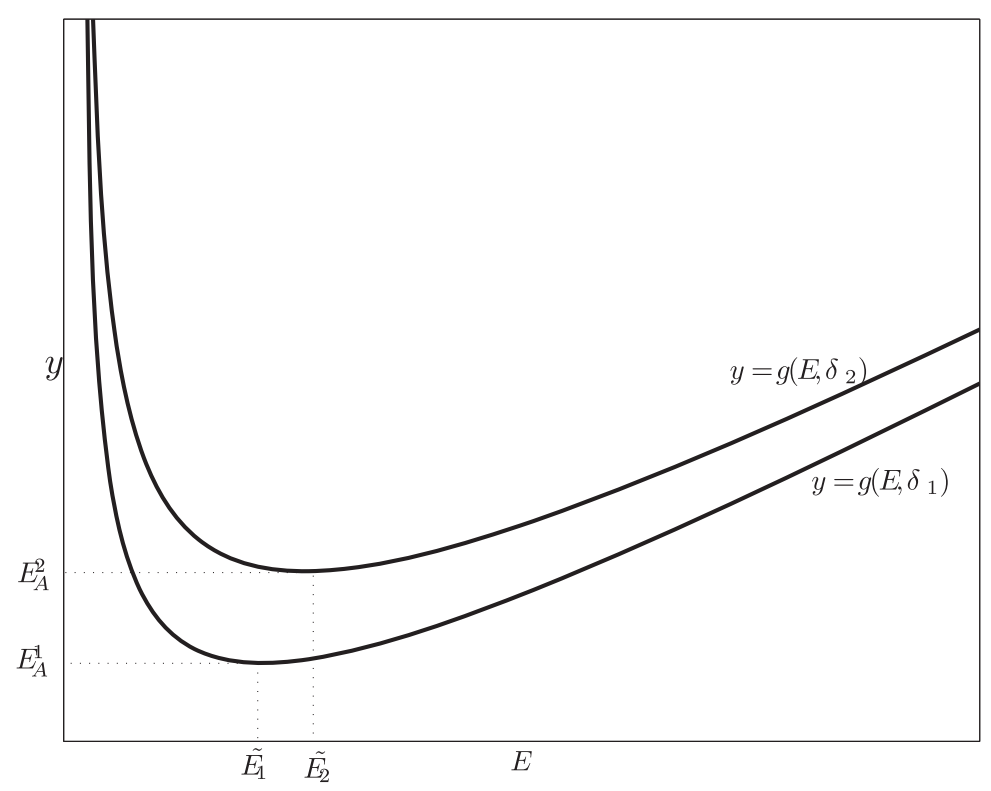

Fig. 1. The graph of $g(E), E>0$, is drawn for values $\delta_{1}<\delta_{2}$ of $\delta$. It is shown that, when $\delta$ increases, both the minimum point $\widetilde{E}$ and the minimum value $E_{A}=g(\widetilde{E})=\frac{2-2 \alpha-\gamma}{1-\alpha-\gamma} \widetilde{E}$ increase.

$$
J^{*}=\left(\begin{array}{ccc}
0 & 0 & \frac{\partial \dot{K}}{\partial L} \\
\frac{\partial \dot{E}}{\partial K} & \frac{\partial \dot{E}}{\partial E} & \frac{\partial \dot{E}}{\partial L} \\
\frac{\partial \dot{L}}{\partial K} & \frac{\partial \dot{L}}{\partial E} & \frac{\partial \dot{L}}{\partial L}
\end{array}\right)
$$

where, by straightforward computations

$$
\begin{aligned}
& \frac{\partial \dot{K}}{\partial L}=\frac{\beta+\varepsilon}{\delta \varepsilon} E^{*}\left(\bar{E}-E^{*}\right) \\
& \frac{\partial \dot{E}}{\partial K}=-\delta \theta \\
& \frac{\partial \dot{E}}{\partial E}=\bar{E}(1-\gamma)-E^{*}(2-\gamma) \\
& \frac{\partial \dot{E}}{\partial L}=-(\beta+\varepsilon) E^{*}\left(\bar{E}-E^{*}\right) \\
& \frac{\partial \dot{L}}{\partial K}=\frac{f\left(L^{*}\right)}{f^{\prime}\left(L^{*}\right)} \frac{\delta \theta}{E^{*}}\left[\frac{\theta(1-\alpha)}{\alpha \eta\left(\bar{E}-E^{*}\right)}-\gamma\right] \\
& \frac{\partial \dot{L}}{\partial E}=\frac{f\left(L^{*}\right)}{f^{\prime}\left(L^{*}\right)} \frac{\gamma}{E^{*}}\left[(1-\gamma)\left(\bar{E}-E^{*}\right)-E^{*}-\frac{\theta}{\eta}\right] \\
& \frac{\partial \dot{L}}{\partial L}=\frac{f\left(L^{*}\right)}{f^{\prime}\left(L^{*}\right)}(\beta+\varepsilon)\left[\frac{\theta(\beta+\varepsilon)}{\varepsilon}-\frac{\theta}{\eta}-\gamma\left(\bar{E}-E^{*}\right)\right]
\end{aligned}
$$

The following theorem holds: 
Theorem 2. If the stationary state is unique with $\alpha+\gamma \geqslant 1$, or, in case of two stationary states, is the one with the larger $E^{*}$, then $J^{*}$ has an odd number of positive eigenvalues; instead, if, in case of two stationary states, $P^{*}$ corresponds to the one with the smaller $E^{*}$, then $J^{*}$ has an odd number of negative eigenvalues.

Proof. By computing $\operatorname{det}\left(J^{*}\right)$, one can check that

$$
\operatorname{sign}\left[\operatorname{det}\left(J^{*}\right)\right]=\operatorname{sign}\left[(2-2 \alpha-\gamma) E^{*}-(1-\alpha-\gamma) \bar{E}\right]
$$

It follows that, when the stationary state is unique and $\alpha+\gamma \geqslant 1$, then $\operatorname{det}\left(J^{*}\right)>0$. Vice versa, when two stationary states exist, implying $\alpha+\gamma<1$, then it follows from (10), by observing Fig. 1, that $\operatorname{det}\left(J^{*}\right)$ has the same sign of $g^{\prime}\left(E^{*}\right)$, which proves the theorem.

It is easy to check that in the non-generic case when a unique stationary state exists under the condition $\alpha+\gamma<1$, then $\operatorname{det}\left(J^{*}\right)=0$ holds and the stationary state is not hyperbolic (in fact, a saddle-node bifurcation occurs). Consequently, if we look for an attracting stationary state, we have to restrict our analysis to the case when, under the assumption $\alpha+\gamma<1$, two stationary states exist, $P_{1}^{*}$ and $P_{2}^{*}$, with $E_{1}^{*}<E_{2}^{*}$ and $K_{1}^{*}<K_{2}^{*}$. We aim to show that, in such a context, $P_{1}^{*}$ can be attracting for suitable values of the parameters. Along the trajectories belonging to the basin of attraction of $P_{1}^{*}$ the over-exploitation of the natural resource drives the economy towards a tragedy of commons scenario.

First of all, if $\alpha+\gamma<1$, a necessary and sufficient condition for the existence of two stationary states is

$$
\bar{E}>E_{A}:=g(\widetilde{E})=\frac{2-2 \alpha-\gamma}{1-\alpha-\gamma} \widetilde{E}
$$

where $\widetilde{E}$ is the only positive value satisfying $g^{\prime}(\widetilde{E})=0$. Straightforward computations yield

$$
E_{A}=(2-2 \alpha-\gamma)\left[\frac{\delta^{1-\alpha}}{(1-\alpha)^{1-\alpha}(1-\alpha-\gamma)^{1-\alpha-\gamma}}\left(\frac{\beta}{\beta+\varepsilon}\right)^{\beta}\left(\frac{\alpha}{\theta}\right)^{\alpha}\right]^{\frac{1}{2-2 \alpha-\gamma}}
$$

Hence $E_{1}^{*}<\widetilde{E}<\frac{1-\alpha-\gamma}{2-2 \alpha-\gamma} \bar{E}$. From now on let us omit the index 1 .

The well-known Routh-Hurwitz Criterion (see Hurwitz [28]) yields that $J^{*}$, the Jacobian matrix at $P^{*}$, has three eigenvalues with negative real part if and only if

$$
\begin{aligned}
& \operatorname{det}\left(J^{*}\right)<0 \\
& \sigma\left(J^{*}\right)=\frac{\partial \dot{E}}{\partial E} \frac{\partial \dot{L}}{\partial L}-\frac{\partial \dot{E}}{\partial L} \frac{\partial \dot{L}}{\partial E}-\frac{\partial \dot{K}}{\partial L} \frac{\partial \dot{L}}{\partial K}>0 \\
& \rho\left(J^{*}\right)=-\sigma\left(J^{*}\right) \cdot \operatorname{trace}\left(J^{*}\right)+\operatorname{det}\left(J^{*}\right)>0
\end{aligned}
$$

The last inequality, in particular, guarantees the non-existence of complex eigenvalues with non-negative real part. In fact, when $\rho\left(J^{*}\right)$ crosses the value 0 , the real part of two complex conjugate eigenvalues changes sign, causing, generically, a Hopf bifurcation.

Remember that the condition (13) is always verified at $P^{*}$ (see (10)). As for the condition (14), we state the following lemma.

\section{Lemma 3. If}

$$
\eta \geqslant \frac{\varepsilon}{\varepsilon+\alpha \beta} \text { and } \bar{E}>E_{B}=\frac{\theta(\beta+\varepsilon)(2-2 \alpha-\gamma)}{\alpha \beta \gamma \eta}
$$

then the condition $\sigma\left(J^{*}\right)>0$ is verified. 
Proof. By recalling (9), straightforward computations lead to

$$
\operatorname{sign}\left[\sigma\left(J^{*}\right)\right]=\operatorname{sign}\left[\left(\frac{\beta+\varepsilon}{\varepsilon}-\frac{1}{\eta}\right)\left(\bar{E}-2 E^{*}\right)-\frac{\theta(1-\alpha)(\beta+\varepsilon)}{\alpha \varepsilon \eta}\right]
$$

So, since $E^{*}<\frac{1-\alpha-\gamma}{2-2 \alpha-\gamma} \bar{E}$, the assumptions of the lemma imply $\sigma\left(J^{*}\right)>0$.

Let us now compute $\operatorname{trace}\left(J^{*}\right)$; observing that $\frac{f\left(L^{*}\right)}{f^{\prime}\left(L^{*}\right)}=\frac{\beta \varepsilon \eta}{(\beta+\varepsilon)[\eta(\beta+\varepsilon)-\beta \varepsilon]}$, we obtain

$$
\operatorname{trace}\left(J^{*}\right)=a\left(\bar{E}-E^{*}\right)-E^{*}+b
$$

where

$$
a:=\frac{\eta[(1-\gamma)(\beta+\varepsilon)-\beta \gamma \varepsilon]-\beta \varepsilon(1-\gamma)}{\eta(\beta+\varepsilon)-\beta \varepsilon}, \quad b:=\frac{\beta \theta[\eta(\beta+\varepsilon)-\varepsilon]}{\eta(\beta+\varepsilon)-\beta \varepsilon}
$$

Then the results of our analysis, aimed at detecting an attracting stationary state, are summarized by the following theorem.

Theorem 4. Let $\alpha+\gamma<1$ and $\bar{E}>E_{A}$, so that system (6) has two stationary states, $P_{1}^{*}$ and $P_{2}^{*}$, with $E_{1}^{*}<E_{2}^{*}$ and $K_{1}^{*}<K_{2}^{*}$. Then there exist values of the parameters for which $P_{1}^{*}$ is a sink, while $P_{2}^{*}$ is a saddle with a two-dimensional stable manifold. Moreover, in such a case, take $\bar{E}$ as a bifurcation parameter. As $\bar{E}$ is increased, $P_{2}^{*}$ does not change its nature (i.e. it remains a saddle with a two-dimensional stable manifold), whereas $P_{1}^{*}$ can undergo one, two or no Hopf bifurcations.

\section{Proof. See Appendix A.}

Notice that the sufficient conditions for local indeterminacy given above depend on the intertemporal elasticity of substitution and can be satisfied both in case $\eta<1$ (i.e. elasticity of substitution greater than 1) and in case $\eta>1$ (i.e. elasticity of substitution lower than 1): in fact, we assumed $\eta \geqslant \frac{\varepsilon}{\varepsilon+\alpha \beta}$. Furthermore, those conditions require that the impact of the production process of output (measured by $\delta$ ) is high enough and/or the subjective discount rate $\theta$ is low enough. Finally, the elasticity $\gamma$ of the production function with respect to the natural capital $E$ must be not too high, that is $\alpha+\gamma<1$, while social returns to scale can be constant or decreasing, that is $\alpha+\beta+\gamma \leqslant 1$.

Fig. 2 shows how the stationary state values of $K$ and $E$ change by varying the value of $\bar{E}$ (the carrying capacity of the environmental resource). The coordinates of $P_{1}^{*}$ are indicated by a bold line if $P_{1}^{*}$ is a sink and by a dash-dot line if it is a saddle with a one-dimensional stable manifold; the coordinates of $P_{2}^{*}$ (which, in the numerical example, is a saddle with a twodimensional stable manifold) are indicated by a dotted line. Notice that a Hopf bifurcation occurs when the parameter $\bar{E}$ crosses the value 0.2 (the bifurcation point is indicated by $\mathrm{H}$ ).

Adopting the same symbology, Fig. 3 draws the stationary states coordinates when varying the parameter $\delta$, which measures the environmental impact of the production process. ${ }^{13}$ Notice that a Hopf bifurcation occurs also in this example and that indeterminacy is observed when $\delta$ is high enough.

13 By observing Fig. 1, it follows that the effect of reducing $\delta$, when $\bar{E}$ is fixed, is qualitatively analogous to that of increasing $\bar{E}$, when $\delta$ is fixed. In fact, as $\delta$ increases, both $\widetilde{E}$ and $E_{A}=g(\widetilde{E})$ increase. 


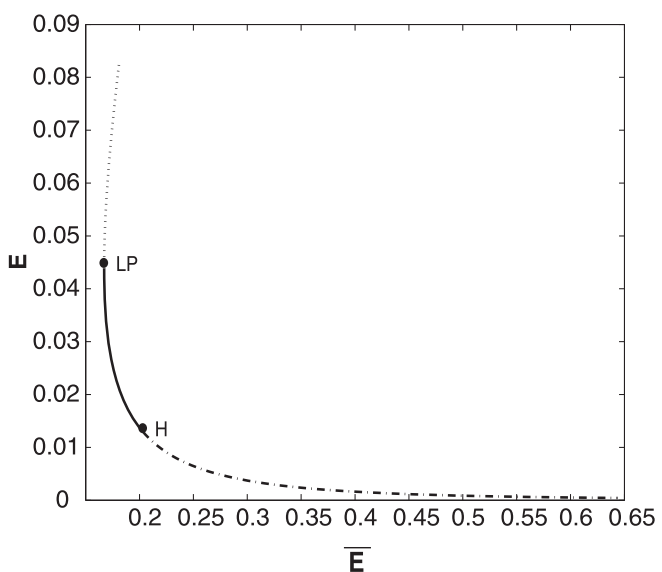

(a)

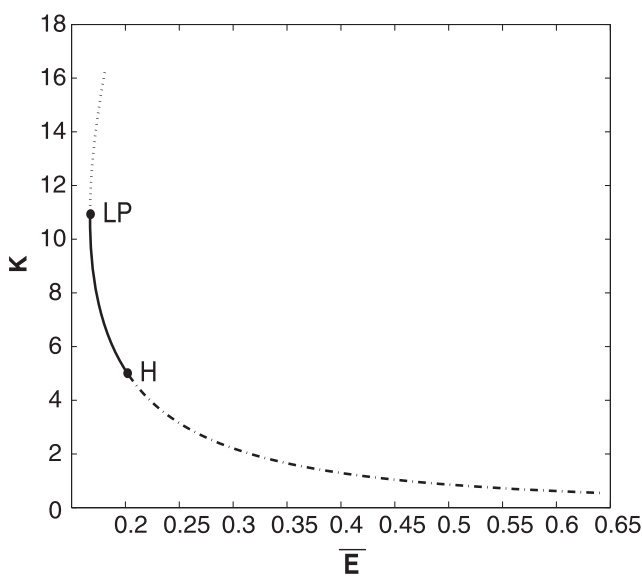

(b)

Fig. 2. The fixed point values of $K$ and $E$, varying $\bar{E}$; parameter values: $\alpha=0.1, \beta=0.8, \gamma=0.58, \delta=0.05, \epsilon=1$, $\eta=1.5, \theta=0.001$.

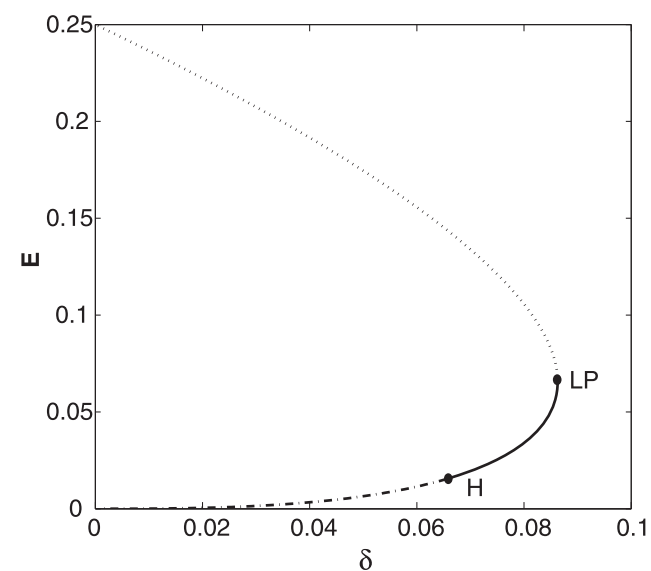

(a)

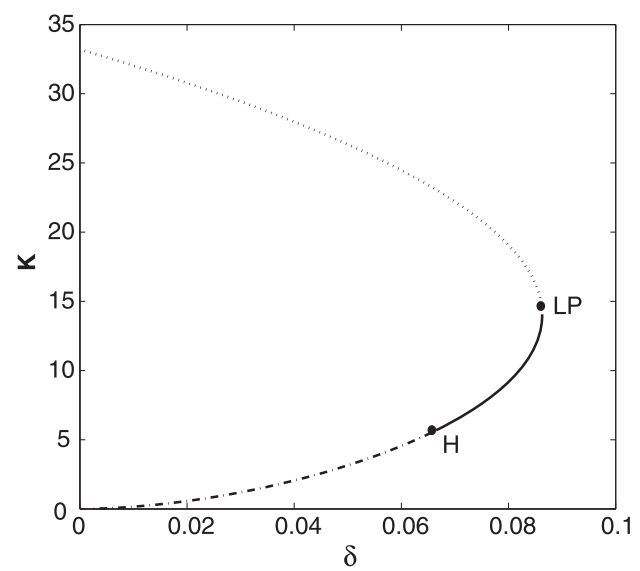

(b)

Fig. 3. The fixed point values of $K$ and $E$, varying $\delta$; parameter values: $\alpha=0.1, \beta=0.8, \gamma=0.58, \epsilon=1, \eta=1.5$, $\theta=0.001, \bar{E}=0.25$.

In Fig. 4, the numerical simulation shows a locally attracting limit cycle around $P_{1}^{*}$ (which has a one-dimensional stable manifold) arisen via the Hopf bifurcation of Fig. 2. In such a case, local indeterminacy occurs, since for every initial point $\left(K_{0}, E_{0}\right)$ close to the projection of the cycle in the plane $(K, E)$, there exists a continuum of initial values $L_{0}$ of $L$ such that the trajectory starting from $\left(K_{0}, E_{0}, L_{0}\right)$ approaches the cycle.

Let us now complete the local analysis by discussing the case $\alpha+\gamma>1$, when the stationary state $P^{*}$ is unique. Since $\operatorname{det}\left(J^{*}\right)>0$ (see Theorem 2 ), $P^{*}$ is not attracting. If, for some value of $\bar{E}$, $\operatorname{trace}\left(J^{*}\right)=a\left(\bar{E}-E^{*}\right)-E^{*}+b<0$, with $a$ and $b$ defined in (16), then $P^{*}$ is a saddle 


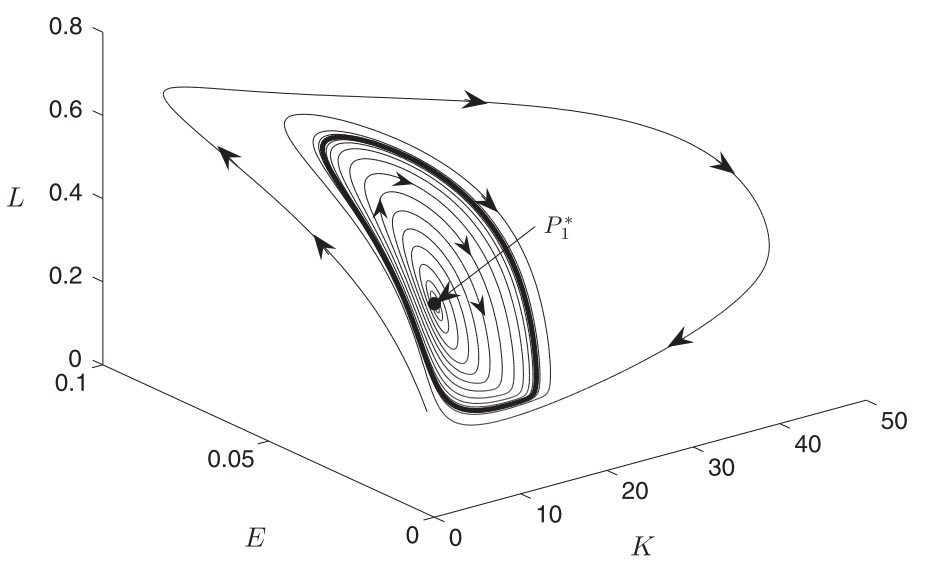

Fig. 4. Locally attracting limit cycle "around" $P_{1}^{*}$; parameter values: $\alpha=0.1, \beta=0.8, \gamma=0.58, \delta=0.05, \epsilon=1$, $\eta=1.5, \theta=0.001, \bar{E}=0.21$.

with a two-dimensional stable manifold. Let us increase $\bar{E}$. Correspondingly $E^{*}$ increases as well. By the equilibrium condition $g\left(E^{*}\right)=\bar{E}$, we can write $\operatorname{trace}\left(J^{*}\right)$ as

$$
\operatorname{trace}\left(J^{*}\right)=r\left(E^{*}\right)^{\frac{\alpha+\gamma-1}{1-\alpha}}-E^{*}+b
$$

with $b>0, \alpha+\gamma-1>0$ and $\operatorname{sign}(r)=\operatorname{sign}(a)$. By the same arguments developed in Theorem 4 , the following theorem is easily proved.

Theorem 5. Let $\alpha+\gamma>1$ and $P^{*}$ denote the only stationary state of system (6). Write trace $\left(J^{*}\right)=a\left(\bar{E}-E^{*}\right)-E^{*}+b$, with a and $b$ defined in (16). Assume trace $\left(J^{*}\right)<0$ for some value of $\bar{E}$ and let $\bar{E}$ increase. Then: if $a \leqslant 0$, no bifurcation occurs and $P^{*}$ remains a saddle with a two-dimensional stable manifold; if $a>0$ and $2 \alpha+\gamma>2$, eventually $P^{*}$ becomes a source and one Hopf bifurcation generically takes place; if $a>0$ and $2 \alpha+\gamma<2, P^{*}$ is a saddle with a two-dimensional stable manifold for sufficiently large values of $\bar{E}$ and Hopf bifurcations are, generically, zero or two.

According to the above theorem, indeterminacy cannot be observed in the context of a unique stationary state: i.e., the stationary state can possess saddle-type stability (two eigenvalues with negative real part) but cannot be a sink.

\section{Global analysis}

In this section, for the sake of convenience in representing the figures, we will change the order of the variables from $(K, E, L)$ into $(E, K, L)$.

In the following we take into consideration the case where, for $\alpha+\gamma<1$, two stationary states exist, $P_{1}^{*}=\left(E_{1}^{*}, K_{1}^{*}, L^{*}\right)$ and $P_{2}^{*}=\left(E_{2}^{*}, K_{2}^{*}, L^{*}\right)$, with $E_{1}^{*}<E_{2}^{*}, K_{1}^{*}<K_{2}^{*}, L^{*}=\frac{\beta}{\beta+\varepsilon}$, and $P_{1}^{*}$ is a sink.

Hence the basin of attraction of $P_{1}^{*}$ can be considered a poverty trap and we wonder if, given a point $P_{0}=\left(E_{0}, K_{0}, L_{0}\right)$ belonging to such a basin, it is possible to modify the initial choice of labor in such a way that the positive semi-trajectory starting from the new point $\widetilde{P}_{0}=\left(E_{0}, K_{0}, \widetilde{L}_{0}\right)$ can tend to the saddle $P_{2}^{*}$ (having a two-dimensional stable manifold). 
In fact we will give a (partially) affirmative answer to the above question (Theorem 10) and, moreover, we will suggest how to conduct numerical experiments aimed at detecting trajectories leading to the desirable equilibrium, i.e. to the saddle $P_{2}^{*}$ (Lemma 7).

These results will be obtained through a partial description of the shape of the saddle-point two-dimensional stable manifold. A full description of such a manifold is usually very difficult or impossible. However, in a three-dimensional system, two-dimensional stable (or unstable) manifolds are separatrices between different regimes of the trajectories. Therefore, if one is able to detect, in a significant region of the phase space (in our case a subregion of the plane $L=L^{*}$ where $\dot{L}>0$ ), a separatrix between two sets of points whose trajectories show different behavior, that may lead to relevant information on the manifold of interest. As we will see, this is in fact our case.

Let us start from the following theorem.

Theorem 6. Consider a point $P_{0}=\left(E_{0}, K_{0}, L_{0}\right), 0<E_{0}<\bar{E}, 0<K_{0}, 0<L_{0}<1$. Then, if $L_{0}$ is small enough, the positive semi-trajectory from $P_{0}$ tends to $(\bar{E}, 0,0)$.

Proof. Assume

$$
L_{0}<\min \left[\frac{\beta}{2(\beta+\varepsilon)},\left[\frac{\frac{\alpha \beta}{2 \varepsilon} K_{0}^{\alpha-1} E_{0}^{\gamma}}{\gamma\left(\bar{E}-E_{0}\right)+\frac{\theta}{\eta}}\right]^{\frac{1}{1-\beta}},\left(\frac{\bar{E}-E_{0}}{\delta K_{0}^{\alpha} E_{0}^{\gamma-1}}\right)^{\frac{1}{\beta}}\right]
$$

By this choice one can compute that, starting from $P_{0}=\left(E_{0}, K_{0}, L_{0}\right), \dot{K}, \dot{L}<0$ and $\dot{E}>0$ in a right interval of $t=0$. Since $\dot{K}<0$ for $L \leqslant L_{0}$ and $\dot{L}<0$ for $E \geqslant E_{0}, K \leqslant K_{0}, L \leqslant L_{0}$, the pattern of the trajectory can change only if $\dot{E}$ turns negative. So, suppose $\dot{E}=0$ at some $\bar{t}>0$. Then

$$
\ddot{E}(\bar{t})=\frac{\partial \dot{E}}{\partial K} \dot{K}+\frac{\partial \dot{E}}{\partial L} \dot{L}>0
$$

since $\frac{\partial \dot{E}}{\partial K}, \dot{K}, \frac{\partial \dot{E}}{\partial L}$ and $\dot{L}$ are all negative at $\bar{t}$. It follows that, for $t>\bar{t}, \dot{E}>0$ again. Hence it is easily checked that the trajectory must tend to the boundary point $(\bar{E}, 0,0)$ and the theorem is proven.

As we said, we want to see if, given a point $P_{0}=\left(E_{0}, K_{0}, L_{0}\right)$ belonging to the basin of attraction of $P_{1}^{*}$ and sufficiently close to $P_{1}^{*}$, it is possible to modify the initial choice of labor in such a way that the positive semi-trajectory starting from the new point $\widetilde{P}_{0}=\left(E_{0}, K_{0}, \widetilde{L}_{0}\right)$ can tend to the saddle $P_{2}^{*}$. To this aim, as suggested by the above theorem we will consider values $\widetilde{L}<L_{0}$. For example, let us start, in order to fix ideas, from $P_{1}^{*}$ itself. Moving downward along the half-line $E=E_{1}^{*}, K=K_{1}^{*}, L<L^{*}$, we cross, as $\underset{\sim}{\sim} \underset{\sim}{\sim}$ in the above theorem, the basin of attraction of $P_{1}^{*}$ at a certain point, say $\widetilde{P}=\left(E_{1}^{*}, K_{1}^{*}, \widetilde{L}\right), \widetilde{L}<L^{*}$. We wonder if the positive semi-trajectory starting from $\widetilde{P}$ tends to the saddle $P_{2}^{*}$. As $K_{1}^{*}<K_{2}^{*}$ and $K(t)$ decreases when $L<L^{*}$, the trajectory from $\widetilde{P}$, in order to tend to $P_{2}^{*}$, must cross, first, the plane $L=L^{*}$. In such a case, that will take place at a point where $K<K_{1}^{*}$ and $\dot{L}>0$.

Furthermore, observe that, being $\widetilde{L}<L^{*}, \dot{E}>0$ at $\widetilde{P}$. Should the trajectory go back, before crossing $L=L^{*}$, to a point where $E=E_{1}^{*}$, at such a point it would be again $\dot{E}>0$, since $K<K_{1}^{*}$ and $L<L^{*}$. So our hypothetical trajectory must cross $L=L^{*}$ at a point where $E>E_{1}^{*}$ and $K<K_{1}^{*}$.

The following lemma allows, precisely, to detect the points with the above described features, i.e. such that $E>E_{1}^{*}, K<K_{1}^{*}, L=L^{*}, \dot{L}>0$, belonging to the stable manifold of $P_{2}^{*}$. 


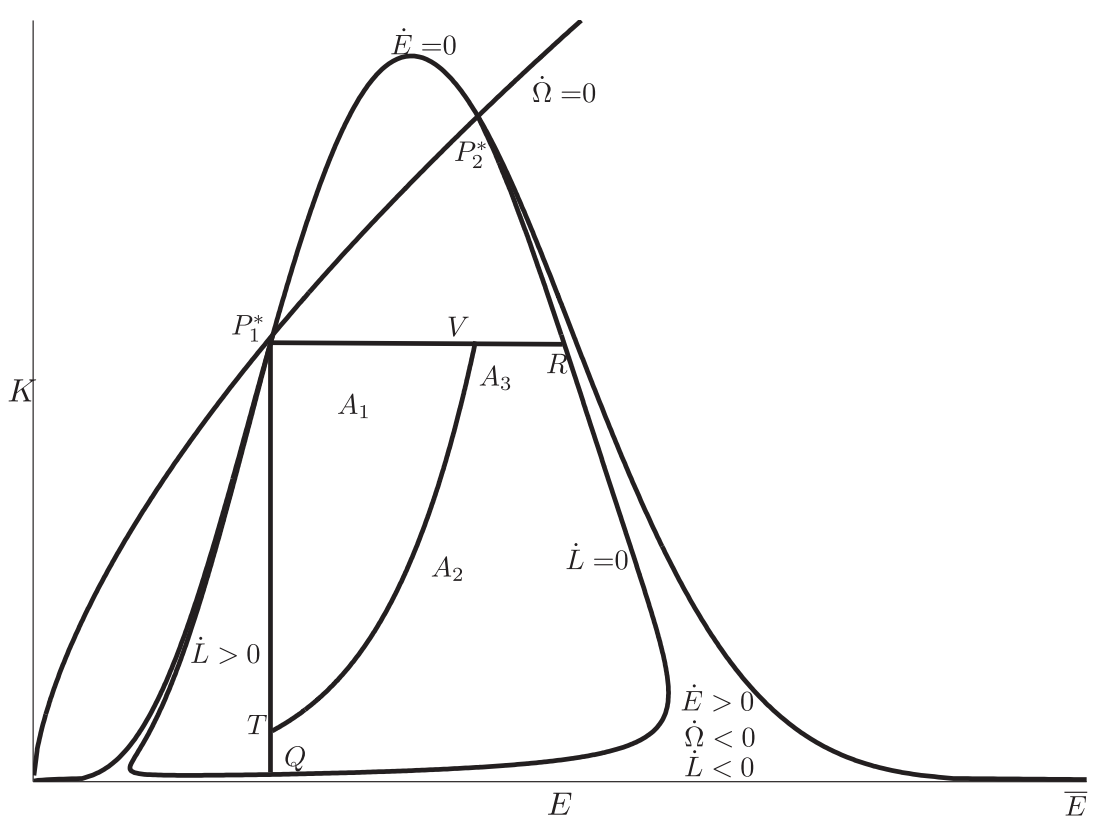

Fig. 5. Configuration of dynamics in the plane $L^{*}=\frac{\beta}{\epsilon+\beta}$; parameter values: $\alpha=0.1, \beta=0.8, \gamma=0.58, \delta=0.05$, $\epsilon=1, \eta=1.5, \theta=0.001, \bar{E}=0.17$.

Lemma 7. Let $\alpha+\gamma<1$ and assume that two equilibria exist, $P_{1}^{*}=\left(E_{1}^{*}, K_{1}^{*}, L^{*}\right)$ and $P_{2}^{*}=$ $\left(E_{2}^{*}, K_{2}^{*}, L^{*}\right), E_{1}^{*}<E_{2}^{*}, K_{1}^{*}<K_{2}^{*}, L^{*}=\frac{\beta}{\beta+\varepsilon}$. Moreover, assume that the conditions of Lemma 3 of the previous section (i.e. $\eta \geqslant \frac{\varepsilon}{\varepsilon+\alpha \beta}, \bar{E}>E_{B}=\frac{\theta(\beta+\varepsilon)(2-2 \alpha-\gamma)}{\alpha \beta \gamma \eta}$ ) are satisfied and that $P_{1}^{*}$ is a sink. Consider, in the plane $L=L^{*}$, the open set

$$
A=\left\{P=\left(E, K, L^{*}\right): E>E_{1}^{*}, K<K_{1}^{*}, \dot{L}(P)>0\right\}
$$

Then A can be partitioned into three non-empty pair-wise disjoint subsets, $A=A_{1} \cup A_{2} \cup A_{3}$, where $A_{1}$ and $A_{2}$ are open, while $A_{3}$ is a unidimensional set belonging to the stable manifold of $P_{2}^{*}$ (see Fig. 5). More precisely:

$A_{1}$ is the subset of points $P \in A$ such that the positive semi-trajectory starting from $P$ crosses $\dot{E}=0$ before crossing again $L=L^{*}$.

$A_{2}$ is the subset of points $Q \in A$ such that the positive semi-trajectory starting from $Q$ crosses again $L=L^{*}$ before crossing $\dot{E}=0$.

Proof. See Appendix B.

An immediate consequence of the previous lemma is the following:

Corollary 8. Let $\alpha+\gamma<1$ and two equilibria, $P_{1}^{*}$ and $P_{2}^{*}$, exist, $P_{1}^{*}$ being a sink. Moreover, assume $\eta \geqslant \frac{\varepsilon}{\varepsilon+\alpha \beta}$ and $\bar{E}>E_{B}=\frac{\theta(\beta+\varepsilon)(2-2 \alpha-\gamma)}{\alpha \beta \gamma \eta}$. Then $P_{2}^{*}$ is a saddle with a two-dimensional stable manifold and the eigenvalues of its Jacobian matrix are all real (one positive and two negative). 
Remark 9. The above corollary suggests that, in the case of two fixed points, whenever $P_{1}^{*}$ is a sink, the eigenvalues at $P_{2}^{*}$ are all real: one positive and two negative (in fact the assumptions of Lemma 3 might be redundant). However it happens that, when $P_{1}^{*}$ is not attracting, $P_{2}^{*}$ can be either a saddle-point with two negative real part complex eigenvalues or a source (all eigenvalues have positive real part).

For example, adopting the above notations, assume, firstly, $E_{A}=E_{C}>\widehat{E}:=$ $\frac{\theta(1-\alpha)(\beta+\varepsilon)(2-2 \alpha-\gamma)}{\alpha \gamma[\eta(\beta+\varepsilon)-\varepsilon]}, a=0$. Then it is easily checked that, if $0<\bar{E}-E_{A} \ll 1, \operatorname{trace}\left[J\left(P_{2}^{*}\right)\right]<$ $0<\operatorname{trace}\left[J\left(P_{1}^{*}\right)\right], \operatorname{det}\left[J\left(P_{1}^{*}\right)\right]<0<\operatorname{det}\left[J\left(P_{2}^{*}\right)\right]$ and $\left|\operatorname{trace}\left[J\left(P_{1,2}^{*}\right)\right]\right| \ll \sigma\left[J\left(P_{1,2}^{*}\right)\right]$ hold. This implies that $P_{1}^{*}$ has one negative and two positive real part complex eigenvalues, while $P_{2}^{*}$ has one positive and two negative real part complex eigenvalues.

Vice versa, let, with the same notations, $E_{C}>E_{A}>\widehat{E}, a=0$. Then, if $0<\bar{E}-E_{A} \ll 1$, both $\operatorname{trace}\left[J\left(P_{1}^{*}\right)\right]$ and $\operatorname{trace}\left[J\left(P_{2}^{*}\right)\right]$ are positive, $\operatorname{det}\left[J\left(P_{1}^{*}\right)\right]<0<\operatorname{det}\left[J\left(P_{2}^{*}\right)\right]$ and $\rho\left[J\left(P_{2}^{*}\right)\right]<0$. Hence in this case $P_{1}^{*}$ has again a one-dimensional stable manifold, while $P_{2}^{*}$ is a source (three positive real part eigenvalues). Moreover, when, posed $E_{A}=\lambda \widehat{E}+(1-\lambda) E_{C}, 0<\lambda<1, \lambda$ is sufficiently small, both $P_{1}^{*}$ and $P_{2}^{*}$ have two complex conjugate eigenvalues.

We are now able to prove our main theorem:

Theorem 10. Given the assumptions of Lemma 7, there exists a neighborhood $N$ of the sink $P_{1}^{*}$, such that, for any $\left(E_{0}, K_{0}, L_{0}\right) \in N$, the half-line $\left\{E=E_{0}, K=K_{0}, L<L_{0}\right\}$ intersects the stable manifold of $P_{2}^{*}$.

Proof. Recalling the notations of Lemma 7, we see that the set $A$, in the plane $L=L^{*}$, looks like (the interior of) a triangoloid with one curvilinear and two straight sides. So, let $P_{1}^{*}, Q$ and $R$ be the vertices of $A$, where $P_{1}^{*}$ is the sink and $Q$ and $R$ are, respectively, the further intersections of $E=E_{1}^{*}$ and $K=K_{1}^{*}$ with the oval $\Gamma=\left\{F(E, K)=0, L=L^{*}\right\}$, i.e. the locus on the plane $L=L^{*}$ where $\dot{L}=0$. We also recall that, at $P_{1}^{*}, \frac{\partial F}{\partial E}>0$ and $\frac{\partial F}{\partial K}<0$. In particular this implies that, at $Q, \frac{\partial F}{\partial K}>0$ (see Fig. 5).

The first step is to prove the following:

Claim. The set $\bar{A}_{3}$ (the closure of $A_{3}$ ), contained in the stable manifold of $P_{2}^{*}$, meets the open segment $\left(Q, P_{1}^{*}\right)$, i.e. the vertical side of $\bar{A}$ (beyond the horizontal side, as shown in the lemma).

For the proof of this claim, which requires rather lengthy but straightforward computations, we refer to Antoci et al. [3].

Actually it can be shown that the arguments utilized in the proof of the claim imply that $\frac{\partial F}{\partial E}<0$ along the whole curvilinear side of $\bar{A}$, i.e. the $\operatorname{arc}[Q, R]$ (see Fig. 5). As a consequence this arc has no intersection with $\bar{A}_{1}$ and thus with $\bar{A}_{3}$ (see the proof of Lemma 7).

Hence, let us indicate by $[T, V]$ a (possibly unique, as in Fig. 5) connected component of $\bar{A}_{3}$, such that $T$ and $V$ belong, respectively, to the open segments $\left(Q, P_{1}^{*}\right)$ and $\left(P_{1}^{*}, R\right)$. It follows that there exist points of $(T, V)$ whose negative (i.e. backward) semi-trajectories intersect the half-plane $H=\left\{E=E_{1}^{*}, L<L^{*}\right\}$. Furthermore, it is easily observed that, if such a semitrajectory intersects $H$ at a point $\left(E_{1}^{*}, \bar{K}, \bar{L}\right)$, with $\bar{L}<L^{*}$ and $\bar{K} \leqslant K_{1}^{*}$, then along it (having exchanged $t$ with $-t$ ) $E$ continues to decrease, while $K$ continues to increase. On the other hand, consider the negative semi-trajectory starting from $V$. Obviously it reaches some half-plane $\left\{K=K_{1}^{*}+\sigma, L<L^{*}\right\}$ with $\sigma>0$. It follows, by the continuous dependence of trajectories on 


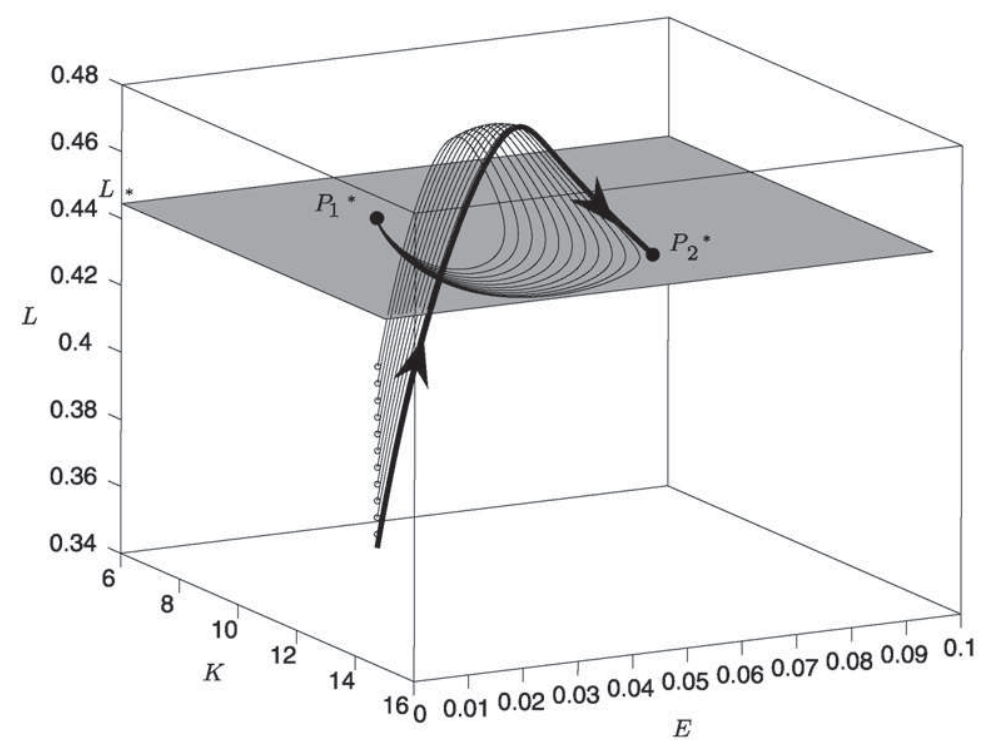

Fig. 6. Global indeterminacy in the space $(E, K, L)$; the trajectory (in bold) approaching $P_{2}^{*}=\left(E_{2}^{*}, K_{2}^{*}, L^{*}=0.44444\right)$ starts from the point $\left(E_{1}^{*}, K_{1}^{*}, L_{0}\right), L_{0} \simeq 0.345910588598$; parameter values: $\alpha=0.1, \beta=0.8, \gamma=0.58, \delta=0.05$, $\epsilon=1, \eta=1.5, \theta=0.001, \bar{E}=0.17$.

initial conditions, that there exists $Z \in(T, V)$ such that its negative semi-trajectory intersects $H$ at some point $U=\left(E_{1}^{*}, K_{1}^{*}+\zeta, \bar{L}\right)$, with $\bar{L}<L^{*}$ and $\zeta>0$.

Therefore, projecting on the plane $L=L^{*}$ the intersections with $H$ of the negative semitrajectories from the $\operatorname{arc}[T, Z] \subset[T, V]$, we get a continuous map $\pi$ from $[T, Z]$ onto a closed segment $\left[T, U^{\prime}\right]$ of the line $\left\{E=E_{1}^{*}, L=L^{*}\right\}$, containing $P_{1}^{*}$. Moreover, a continuity argument, again, implies that the negative semi-trajectories from $[T, Z]$ also cross any half-plane $\{E=$ $\left.E_{1}^{*}-v, L<L^{*}\right\}$, if $v>0$ is sufficiently small.

This concludes the proof of the theorem.

Hence, under the assumptions of the theorem, for any initial point $\left(E_{0}, K_{0}\right)$ sufficiently close to $\left(E_{1}^{*}, K_{1}^{*}\right)$, there exists a continuum of initial values $L_{0}^{1}$ such that the trajectory starting from $\left(E_{0}, K_{0}, L_{0}^{1}\right)$ approaches $P_{1}^{*}$, and a locally unique value $L_{0}^{2}$ such that the trajectory starting from $\left(E_{0}, K_{0}, L_{0}^{2}\right)$ converges to $P_{2}^{*}$. So global indeterminacy occurs, since, from the initial position $\left(E_{0}, K_{0}\right)$, the economy may follow one of the trajectories belonging to the basin of attraction of the poverty trap $P_{1}^{*}$ but it may also follow a trajectory lying on the stable manifold of the stationary state $P_{2}^{*}$.

In Fig. 6 a numerical simulation is shown. The starting points of the trajectories are chosen along the half-line $\left\{E=E_{1}^{*}, K=K_{1}^{*}, L<L^{*}\right\}$. The trajectory in bold lies on the stable manifold of $P_{2}^{*}$ and consequently converges to $P_{2}^{*}$, while all the others approach $P_{1}^{*}$. Notice that some trajectories approaching $P_{1}^{*}$ are characterized by an initial phase where the values of $K$ and $L$ are higher than along the trajectory converging to $P_{2}^{*}$; however, the higher values of $K$ and $L$ give rise to over-exploitation of the natural resource and the consequent reduction of the stock $E$ drives the economy towards the undesirable equilibrium $P_{1}^{*}$, where the values of $K$ and $E$ are lower than in $P_{2}^{*}$. 


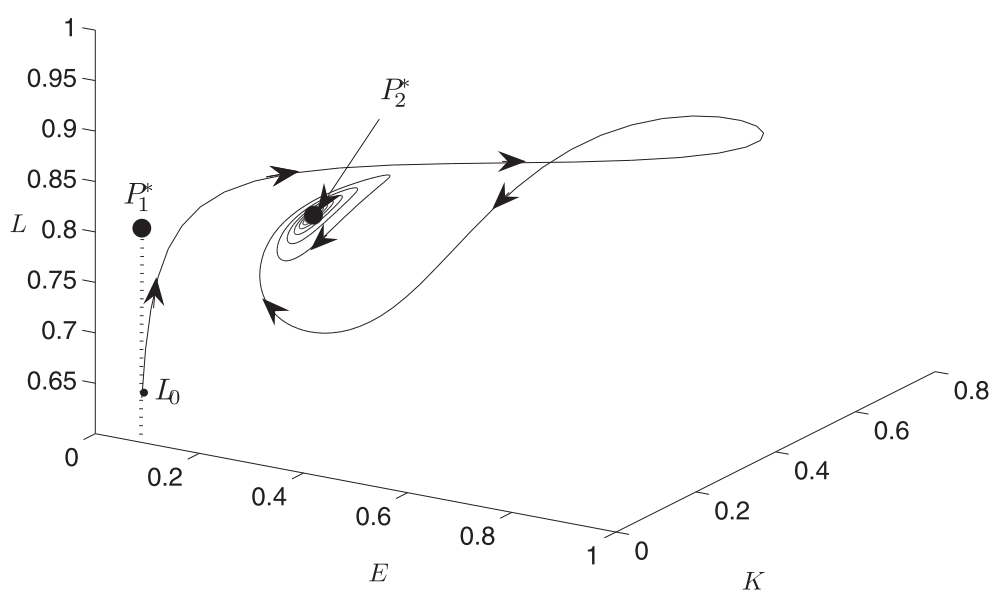

Fig. 7. Trajectory spiraling toward $P_{2}^{*}$ starting from the point $\left(E_{1}^{*}, K_{1}^{*}, L_{0} \simeq 0.6316546\right)$; parameter values: $\alpha=\theta=$ $0.19, \beta=\gamma=0.8, \epsilon=0.2, \eta=\frac{4}{9}, \delta=12.8620934285269, \bar{E}=10.75$.

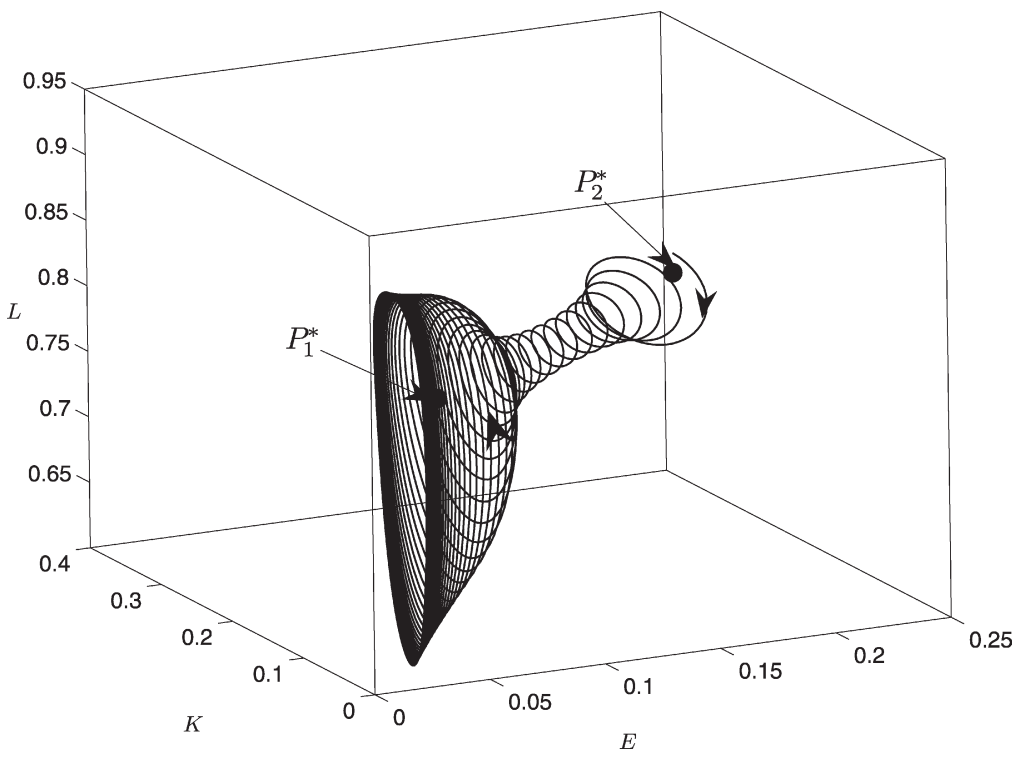

Fig. 8. Starting from the point $\left(E_{2}^{*}, K_{2}^{*}, L_{0}=0.815\right)$, "very close" to $P_{2}^{*}=\left(E_{2}^{*}, K_{2}^{*}, L^{*}=0.8\right)$, the trajectory approaches the attracting limit cycle surrounding $P_{1}^{*}$; the parameter values are those used in the simulation showed in Fig. 7.

In our model, global indeterminacy can occur even if the assumptions of Lemma 7 are not satisfied, as the trajectories drawn in Figs. 7-9 suggest.

In Figs. 7 and $8, P_{1}^{*}=\left(E_{1}^{*}, K_{1}^{*}, L^{*}\right)$ has one negative and two positive real part complex eigenvalues, while $P_{2}^{*}$ has one positive and two negative real part complex eigenvalues. Numerical simulations suggest the existence of an attracting limit cycle surrounding $P_{1}^{*}$. In Fig. 7 the point $\left(E_{1}^{*}, K_{1}^{*}, L_{0}\right)$, lying on the half-line $\left\{E=E_{1}^{*}, K=K_{1}^{*}, L<L^{*}\right\}$, appears to belong to the stable manifold of $P_{2}^{*}$ (i.e. the trajectory starting from it approaches $P_{2}^{*}$ ). Using the same 


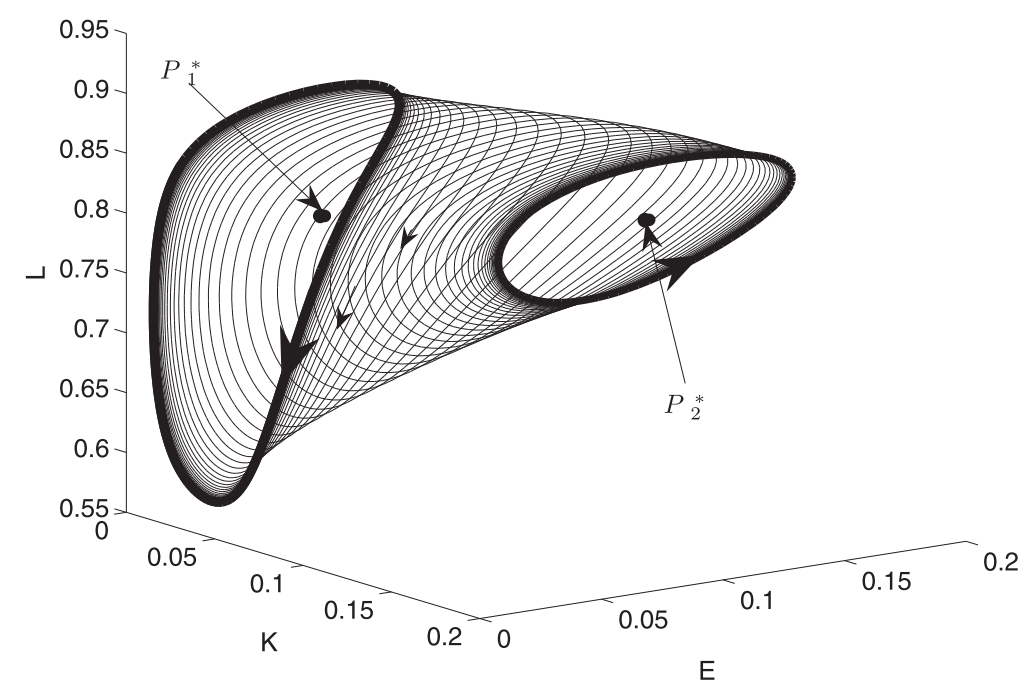

Fig. 9. Two limit cycles: an attractive one "around" $P_{1}^{*}$ and another one "around" $P_{2}^{*}$; parameter values: $\alpha=\theta=0.19$, $\beta=\gamma=0.8, \epsilon=0.2, \eta=\frac{4}{9}, \delta=8.42228, \bar{E}=7.06$.

parameter values of Fig. 7, the simulation in Fig. 8 shows a trajectory starting from a point on the half-line $\left\{E=E_{2}^{*}, K=K_{2}^{*}, L>L^{*}\right\}$, very close to the saddle-point $P_{2}^{*}$, which approaches the attracting limit cycle surrounding $P_{1}^{*}$.

Fig. 9 shows the occurrence of two limit cycles. In this case $P_{1}^{*}$ has one negative and two positive real part complex eigenvalues, while $P_{2}^{*}$ is a source (three positive real part eigenvalues). The simulation shows an attracting limit cycle surrounding $P_{1}^{*}$ and a not-attracting one arisen around $P_{2}^{*}$ through a Hopf bifurcation and endowed with a two-dimensional stable manifold, which bounds, as a separatrix, the basin of the attracting limit cycle.

\section{Appendix A. Proof of Theorem 4}

First of all, let $E_{C}:=\frac{b(2-2 \alpha-\gamma)}{1-\alpha-\gamma}$, where $b$ is defined in (16). Assuming $\eta \geqslant \frac{\varepsilon}{\varepsilon+\alpha \beta}$, recall the expressions of $E_{A}(12)$ and $E_{B}(15)$. It is easily checked that, for $\theta$ sufficiently small or/and $\delta$ sufficiently large,

$$
E_{A}>\max \left(E_{B}, E_{C}\right)
$$

Let $\bar{E}$ be sufficiently close to $E_{A}, 0<\bar{E}-E_{A} \ll 1$. Then, if

$$
\begin{aligned}
& a \leqslant 0 \quad \text { or } 0<a<\frac{1-\alpha-\gamma}{1-\alpha} \text { and } E_{A}>\frac{1-\alpha-\gamma}{1-\alpha-\gamma-a(1-\alpha)} E_{C} \\
& \operatorname{trace}\left(J^{*}\right)<0
\end{aligned}
$$

both at $P_{1}^{*}$ and $P_{2}^{*}$.

Furthermore, since $\operatorname{det}\left(J^{*}\right)=0$ for $E^{*}=E_{A}$, also $\rho\left(J^{*}\right)$, in addition to $\sigma\left(J^{*}\right)$, is positive. It follows that $J^{*}$ has three eigenvalues with negative real part at $P_{1}^{*}$ and two with negative real part and one positive eigenvalues at $P_{2}^{*}$, which proves the first statement of the theorem. 
Now, let $\bar{E}$ increase, ceteris paribus. Since $E_{2}^{*}$ increases and $\bar{E}-E_{2}^{*}=\delta\left(\frac{\beta}{\beta+\varepsilon}\right)^{\frac{\beta}{1-\alpha}}\left(\frac{\alpha}{\theta}\right)^{\frac{\alpha}{1-\alpha}} \times$ $\left(E_{2}^{*}\right)^{\frac{\alpha+\gamma-1}{1-\alpha}}$ decreases, it follows that, no matter what the sign of $a$ is, $P_{2}^{*}$ remains a saddle with a two-dimensional stable manifold.

Instead, since $E_{1}^{*}$ decreases as $\bar{E}$ increases, $P_{1}^{*}$ undergoes (generically) one Hopf bifurcation if $a \geqslant 0$ : the real part of two complex eigenvalues turns from negative into positive and eventually $\operatorname{trace}\left(J^{*}\right)$ becomes positive.

On the contrary, if $a<0$, it happens that, when $\bar{E}$ is large enough, $\operatorname{trace}\left(J^{*}\right)<0$, while $\sigma\left(J^{*}\right)$ and $\rho\left(J^{*}\right)$ are both positive: in fact it is easily checked that $\rho\left(J^{*}\right)$ is a second degree polynomial in $\bar{E}$ with a positive coefficient of $\bar{E}^{2}$. So $P_{1}^{*}$ is a sink for large values of $\bar{E}$. As to the way of detecting, in such a case, possible Hopf bifurcations, see Antoci et al. [3].

\section{Appendix B. Proof of Lemma 7}

We start by describing the region of $L=L^{*}$ where $\dot{L}>0$. First of all we check that the curve $\dot{L}=0$ lying in the plane $L=L^{*}$, say $\Gamma$, is crossed at most twice by each line $E=E_{0}$ or $K=K_{0}$. In fact, set first $E=E_{0}$ and $L=L^{*}=\frac{\beta}{\beta+\varepsilon}$ in the equation $\dot{L}=0$ (see (6)). Then the solutions, in $K$, are given by the intersections of the horizontal line, say $Y=\gamma\left(\bar{E}-E_{0}\right)+\frac{\theta}{\eta}$, with the graph of a function of the type $Y=a K^{\alpha}+b K^{\alpha-1}, a, b>0$, having precisely one minimum. Analogously, set in $\dot{L}=0, K=K_{0}$ and $L=L^{*}$. Now the solutions of the equation, in $E$, are given by the intersections of the line with negative slope $Y=\gamma(\bar{E}-E)+\frac{\theta}{\eta}$ with the graph of a function of the type $Y=c E^{\gamma}+d E^{\gamma-1}, c, d>0$, having precisely one minimum at $E=E_{m}>0$ and one inflection at $E=E_{i}>E_{m}$. In fact, recalling $\alpha+\gamma<1$, it can be checked that $\Gamma$ is an oval contained in a rectangle $\left[E^{\prime}, E^{\prime \prime}\right] \times\left[K^{\prime}, K^{\prime \prime}\right]$, where $0<E^{\prime}<E_{1}^{*}<E_{2}^{*}<E^{\prime \prime}$, $0<K^{\prime}<K_{1}^{*}<K_{2}^{*}<K^{\prime \prime}$ (but it may happen that $E^{\prime \prime} \geqslant \bar{E}$ ). Thus the region of $L=L^{*}$ where $\dot{L}>0$ is the open region, say $C$, bounded by $\Gamma$.

More specifically let us consider the sub-region $B$ of $C$, lying below the line joining $P_{1}^{*}$ and $P_{2}^{*}$, where $K$ is taken as the vertical coordinate. Again we can check that, at a point $P=$ $\left(E, K, L^{*}\right) \in B, \dot{E}>0$ and $\dot{\Omega}<0$ (i.e. $\alpha K^{\alpha-1}\left(L^{*}\right)^{\beta} E^{\gamma}>\theta$ ), as shown in Fig. 5 .

Moreover, we claim that the conditions of Lemma 3 of the previous section (in particular $\bar{E}>E_{B}$ ), which are supposed to hold, imply that the tangent line to $\Gamma$ at $P_{1}^{*}$ has a positive slope. In fact, write the equation of $\Gamma$ on $L=L^{*}$

$$
\text { I) } \quad F(E, K):=\gamma\left[\bar{E}-E-\delta K^{\alpha}\left(L^{*}\right)^{\beta} E^{\gamma-1}\right]+\frac{1}{\eta}\left[\theta-\alpha K^{\alpha-1}\left(L^{*}\right)^{\beta} E^{\gamma}\right]=0
$$

and compute $\frac{\partial F}{\partial E}$ and $\frac{\partial F}{\partial K}$ at $\left(E_{1}^{*}, K_{1}^{*}\right)$. After easy steps, we obtain

$$
\frac{\partial F}{\partial E}=\frac{\gamma}{E_{1}^{*}}\left[(1-\gamma)\left(\bar{E}-E_{1}^{*}\right)-E_{1}^{*}-\frac{\theta}{\eta}\right]
$$

By recalling $E_{1}^{*}<\frac{1-\alpha-\gamma}{2-2 \alpha-\gamma} \bar{E}$ and the expression of $E_{B}$, it can be checked that our assumptions imply $\frac{\partial F}{\partial E}>0$. Analogously it is proved that $\frac{\partial F}{\partial K}<0$ at $\left(E_{1}^{*}, K_{1}^{*}\right)$. So the claim follows.

Finally, let us consider $A \subset B$ and $A_{1}, A_{2} \subset A$, as defined in the statement of the lemma. First of all, let us see that $A_{1}$ and $A_{2}$ are non-empty.

In fact, if $P=\left(E, K, L^{*}\right) \in A$ belongs to the basin of attraction of $P_{1}^{*}$ and is sufficiently close to $P_{1}^{*}$, then the positive semi-trajectory starting from $P$ must remain close to $P_{1}^{*}$, because $P_{1}^{*}$, being a sink, is in particular Lyapunov stable. However, since $E>E_{1}^{*}$, this implies (see again 
Fig. 5) that the positive semi-trajectory from $P$ crosses $\dot{E}=0$ before possibly crossing again $L=L^{*}$.

As to $A_{2}$, consider the intersections of $\Gamma$ with the line $K=K_{1}^{*}$ (on $L=L^{*}$ ). One intersection is, of course, $P_{1}^{*}$, while the other is a point $R=\left(\widetilde{E}, K_{1}^{*}, L^{*}\right)$ with $\widetilde{E}>E_{1}^{*}$. Then $\dot{L}(R)=0$ and we want to show that $\ddot{L}(R)<0$. In fact, $E_{1}^{*}$ and $\widetilde{E}$ are the solutions of the equation

$$
G(E)=F\left(E, K_{1}^{*}\right)=0
$$

Since we have seen that $G^{\prime}\left(E_{1}^{*}\right)=\frac{\partial F}{\partial E}\left(E_{1}^{*}, K_{1}^{*}\right)>0$ and is easily checked that $G^{\prime}(E)$ has only one zero in the interval $\left[E_{1}^{*}, \widetilde{E}\right]$ (corresponding to the intersection of one increasing and one decreasing graph), it follows that $G^{\prime}(\widetilde{E})<0$.

Hence

$$
\ddot{L}(R)=G^{\prime}(\widetilde{E}) \dot{E}(R)<0
$$

This means that, along the trajectory through $R, L(t)$ has a relative maximum at $R$. Therefore, by the continuous dependence of trajectories on initial conditions (see Arnold [5]), it follows that positive semi-trajectories from points of $A$ sufficiently close to $R$ cross again $L=L^{*}$ near $R$, and thus before reaching $\dot{E}=0$ (see Fig. 5).

Once we have proved that $A_{1}$ and $A_{2}$ are non-empty, their openness is just a consequence of the continuous dependence of trajectories on initial conditions, as in the definitions of $A_{1}$ and $A_{2}$ we have required trajectories to cross, and not merely touch, respectively, $\dot{E}=0$ and $L=L^{*}$.

So $A_{1}$ and $A_{2}$ are two non-empty disjoint open subsets of $A$ (in the plane $L=L^{*}$ ). But, since $A$ is connected, there exist points of $A$ which don't lie either in $A_{1}$ or $A_{2}$. Let $A_{3}=A-A_{1} \cup A_{2}$. Clearly $A_{3}$ has no isolated point.

Consider, now, the positive semi-trajectory starting from some $Q_{0} \in A_{3}$. Along it $E$ initially increases. May the trajectory cross at the same instant of time, say $t_{0}>0$, both $\dot{E}=0$ and $L=L^{*}$ ? In such a case, as $t_{0}$ is finite, $\dot{E}\left(t_{0}\right)=\dot{K}\left(t_{0}\right)=0$, while $\dot{L}\left(t_{0}\right)<0$. Hence

$$
\ddot{E}\left(t_{0}\right)=\frac{\partial \dot{E}}{\partial L} \dot{L}\left(t_{0}\right)>0
$$

Therefore $E(t)$ has a (relative) minimum at $t_{0}$ and thus cannot increase for $t<t_{0}$.

So, along the positive semi-trajectory from $Q_{0}, E$ keeps increasing and so does $K$, as $L>L^{*}$. However $L$ cannot tend to 1 as $t \rightarrow+\infty$, since, along any positive semi-trajectory on the invariant plane $L=1, E \rightarrow 0$, as it is easily checked. This implies that, if along a trajectory $L$ and $E$ both increase and $L$ approaches 1 , at a certain point the trajectory must cross the surface $\dot{E}=0$.

As a conclusion the positive semi-trajectory starting from $Q_{0} \in A_{3}$ must tend to the saddle $P_{2}^{*}$.

It follows that $P_{2}^{*}$ has a two-dimensional stable manifold, that $A_{3}$ is unidimensional and finally that trajectories tending to $P_{2}^{*}$ do not spiral.

\section{References}

[1] A. Antoci, M. Galeotti, P. Russu, Consumption of private goods as substitutes for environmental goods in an economic growth model, Nonlinear Analysis: Modelling and Control 10 (2005) 3-34.

[2] A. Antoci, M. Sodini, Indeterminacy, bifurcations and chaos in an overlapping generations model with negative environmental externalities, Chaos, Solitons and Fractals 42 (2009) 1439-1450.

[3] A. Antoci, M. Galeotti, P. Russu, Over-exploitation of open-access natural resources and global indeterminacy in an economic growth model, DiMaD working paper no. 2009-05, Dipartimento di Matematica per le Decisioni, Firenze, 2009. 
[4] A. Antoci, M. Sodini, A. Naimzada, Bifurcations and chaotic attractors in an overlapping generations model with negative environmental externalities, in: G. Bischi, C. Chiarella, L. Gardini (Eds.), Nonlinear Dynamics in Economics, Finance and Social Sciences, Springer-Verlag, Amsterdam, 2010, pp. 39-53.

[5] V.I. Arnold, Ordinary Differential Equations, MIT Press, Cambridge, 1978.

[6] A.D. Ayong Le Kama, Sustainable growth, renewable resources and pollution, J. Econ. Dynam. Control 25 (2001) 1911-1918.

[7] J. Benhabib, R.E. Farmer, Indeterminacy and increasing returns, J. Econ. Theory 63 (1994) 19-41.

[8] J. Benhabib, K. Nishimura, Indeterminacy and sunspots with constant returns, J. Econ. Theory 81 (1998) 58-96.

[9] J. Benhabib, K. Nishimura, Indeterminacy arising in multi-sector economies, Japanese Econ. Rev. 50 (1999) 485506.

[10] J. Benhabib, R.E. Farmer, Indeterminacy and sunspots in macroeconomics, in: J.B. Taylor, M. Woodford (Eds.), Handbook of Macroeconomics, North-Holland, Amsterdam, 1999, pp. 387-448.

[11] J. Benhabib, S. Eusepi, The design of monetary and fiscal policy: A global perspective, J. Econ. Theory 123 (2005) $40-73$.

[12] J. Benhabib, K. Nishimura, T. Shigoka, Bifurcation and sunspots in the continuous time equilibrium model with capacity utilization, Int. J. Econ. Theory 4 (2008) 337-355.

[13] R.L. Bennet, R.E. Farmer, Indeterminacy with non-separable utility, J. Econ. Theory 93 (2000) 118-143.

[14] P. Berck, J.M. Perloff, An open-access fishery with rational expectations, Econometrica 52 (1984) 489-506.

[15] G.I. Bischi, F. Lamantia, Harvesting dynamics and unprotected areas, J. Econ. Behav. Organ. 62 (2007) $348-370$.

[16] M. Boldrin, A. Rustichini, Indeterminacy of equilibria in models with infinitely-lived agents and external effects, Econometrica 62 (1994) 323-342.

[17] P. Brito, A. Venditti, Local and global indeterminacy in two-sector models of endogenous growth, J. Math. Econom. (2010), doi:10.1016/j.jmateco.2010.08.003.

[18] G.M. Brown, Renewable natural resource management and use without markets, J. Econ. Lit. 38 (2000) $875-914$.

[19] J.P. Caulkins, G. Feichtinger, M. Johnson, G. Tragler, Y. Yegorov, Skiba thresholds in a model of controlled migration, J. Econ. Behav. Organ. 57 (2005) 490-508.

[20] J.P. Caulkins, R.F. Hartl, P.M. Kort, G. Feichtinger, Explaining fashion cycles: Imitators chasing innovators in product space, J. Econ. Dynam. Control 31 (2007) 1535-1556.

[21] B.-L. Chen, S.-F. Lee, Congestible public goods and local indeterminacy: A two-sector endogenous growth model, J. Econ. Dynam. Control 31 (2007) 2486-2518.

[22] T. Coury, Y. Wen, Global indeterminacy in locally determinate real business cycle models, Int. J. Econ. Theory 5 (2009) 49-60.

[23] S. D'Alessandro, Non-linear dynamics of population and natural resources: The emergence of different patterns of development, Ecolog. Econ. 62 (2007) 473-481.

[24] W.D. Dechert, K. Nishimura, A complete characterization of optimal growth paths in an aggregated model with a non-concave production function, J. Econ. Theory 31 (1983) 332-354.

[25] L. Elíasson, S. Turnovsky, Renewable resources in an endogenously growing economy: Balanced growth and transitional dynamics, J. Environ. Econ. Manage. 48 (2004) 1018-1049.

[26] H.S. Gordon, The economic theory of a common property resource: The fishery, J. Polit. Economy 63 (1954) 124142

[27] R.F. Hartl, P.M. Kort, G. Feichtinger, F. Wirl, Multiple equilibria and thresholds due to relative investment costs, J. Optim. Theory Appl. 123 (2004) 49-82.

[28] A. Hurwitz, On the conditions under which an equation has only roots with negative real parts, in: R. Bellman, R. Kalaba (Eds.), Selected Papers on Mathematical Trends in Control Theory, Dover Publications, New York, 1964.

[29] J.-I. Itaya, Can environmental taxation stimulate growth? The role of indeterminacy in endogenous growth models with environmental externalities, J. Econ. Dynam. Control 32 (2008) 1156-1180.

[30] L. Karp, T. Paul, Indeterminacy with environmental and labor dynamics, Struct. Change Econ. Dynam. 18 (2007) $100-119$.

[31] P. Krugman, History versus expectations, Quart. J. Econ. 106 (1991) 651-667.

[32] R.E. López, The policy roots of socioeconomic stagnation and environmental implosion: Latin America 1950-2000, World Devel. 31 (2003) 259-280.

[33] R.E. López, Structural change, poverty and natural resource degradation, in: G. Atkinson, S. Dietz, E. Neumayer (Eds.), Handbook of Sustainable Development, Edwards Elgar, Cheltenham, UK/Northhampton, MA, USA, 2007.

[34] R.E. López, G. Anríquez, S. Gulati, Structural change and sustainable development, J. Environ. Econ. Manage. 53 (2007) 307-322

[35] K. Matsuyama, Increasing returns, industrialization, and indeterminacy of equilibrium, Quart. J. Econ. 106 (1991) 617-650. 
[36] P. Mattana, K. Nishimura, T. Shigoka, Homoclinic bifurcation and global indeterminacy of equilibrium in a twosector endogenous growth model, Int. J. Econ. Theory 5 (2009) 25-47.

[37] S.F. McWhinnie, The tragedy of commons in international fisheries: An empirical examination, J. Environ. Econ. Manage. 57 (2009) 321-333.

[38] Q. Meng, C.K. Yip, On indeterminacy in a one-sector models of the business cycle with factor generated externalities, J. Macroecon. 30 (2008) 97-110.

[39] K. Mino, Non-separable utility function and indeterminacy of equilibrium in a model with human capital, Econ. Letters 62 (1999) 311-317.

[40] K. Mino, K. Nishimura, K. Shimomura, P. Wang, Equilibrium dynamics in discrete-time endogenous growth models with social constant returns, Econ. Theory 34 (2008) 1-23.

[41] K. Nishimura, K. Shimomura, Indeterminacy in a dynamic small open economy, J. Econ. Dynam. Control 27 (2002) 271-281.

[42] K. Nishimura, C. Nourry, A. Venditti, Indeterminacy in aggregate models with small externalities: An interplay between preferences and technology, J. Nonlinear Convex Anal. 10 (2009) 279-298.

[43] R. Pérez, J. Ruiz, Global and local indeterminacy and optimal environmental public policies in an economy with public abatement activities, Econ. Modelling 24 (2007) 431-452.

[44] R. Perli, Indeterminacy, home production and the business cycles, J. Monet. Econ. 41 (1998) 105-125.

[45] P. Pintus, D. Sands, R. de Vilder, On the transition from local regular to global irregular fluctuations, J. Econ. Dynam. Control 24 (2000) 247-272.

[46] X. Raurich-Puigdevall, Global indeterminacy in an endogenous-growth model with public capital, J. Econ. 71 (2000) 255-280.

[47] M.B. Schaefer, Some considerations of population dynamics and economics in relation to the management of marine fisheries, J. Fish. Res. Board Canada 14 (1957) 669-681.

[48] S.P. Sethi, Nearest feasible paths in optimal control problems: Theory, examples and counterexamples, J. Optim. Theory Appl. 23 (1977) 563-579.

[49] A. Skiba, Optimal growth with a convex-concave production function, Econometrica 46 (1978) 527-540.

[50] F.O.O. Wagener, Skiba points and heteroclinic bifurcations, with applications to the shallow lake system, J. Econ. Dynam. Control 27 (2003) 1533-1561.

[51] F.O.O. Wagener, Skiba points for small discount rates, J. Optim. Theory Appl. 128 (2006) 261-277.

[52] F. Wirl, The cyclical exploitation of renewable resource stocks may be optimal, J. Environ. Econ. Manage. 29 (1995) $252-261$.

[53] F. Wirl, Stability and limit cycles in one-dimensional dynamic optimizations of competitive agents with a market externality, J. Evol. Econ. 7 (1997) 73-89.

[54] F. Wirl, Sustainable growth, renewable resources and pollution: thresholds and cycles, J. Econ. Dynam. Control 28 (2004) 1149-1157.

[55] F. Wirl, G. Feichtinger, History dependence in concave economies, J. Econ. Behav. Organ. 57 (2005) $390-407$.

[56] Y. Zhang, Does the utility function matter for indeterminacy in a two-sector small open economy? Ann. Econ. Finance 9 (2008) 61-71. 